\title{
Searching the landscape of flux vacua with genetic algorithms
}

\author{
Alex Cole, Andreas Schachner and Gary Shiu \\ Department of Physics, University of Wisconsin, \\ 1150 University Avenue, Madison, WI 53706, U.S.A. \\ E-mail: acole4@wisc.edu, schachner@thphys.uni-heidelberg.de, \\ shiu@physics.wisc.edu
}

ABSTRACT: In this paper, we employ genetic algorithms to explore the landscape of type IIB flux vacua. We show that genetic algorithms can efficiently scan the landscape for viable solutions satisfying various criteria. More specifically, we consider a symmetric $T^{6}$ as well as the conifold region of a Calabi-Yau hypersurface. We argue that in both cases genetic algorithms are powerful tools for finding flux vacua with interesting phenomenological properties. We also compare genetic algorithms to algorithms based on different breeding mechanisms as well as random walk approaches.

KEYWORDS: Superstring Vacua, Flux compactifications

ArXiv EPrint: 1907.10072 


\section{Contents}

1 Introduction 1

2 Flux compactifications $\quad 4$

3 Genetic algorithms $\quad 6$

3.1 Generalities 6

$\begin{array}{lll}3.2 & \text { Genetic algorithms for flux vacua } & 7\end{array}$

$\begin{array}{lll}3.3 & \text { Schemata and fitness distance correlation } & 9\end{array}$

4 Calabi-Yau hypersurface $\quad 11$

4.1 Expansion around the conifold locus 11

$\begin{array}{ll}4.2 & \text { Analyzing different mutation rates } \\ & 12\end{array}$

$\begin{array}{ll}4.3 \text { Genetic algorithm dynamics } & 13\end{array}$

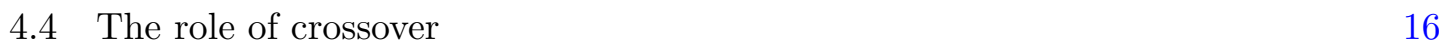

$\begin{array}{ll}\text { 4.5 Comparing different breeding mechanisms } & 17\end{array}$

$\begin{array}{lll}\text { 4.6 Comparison to a Metropolis algorithm } & 18\end{array}$

5 Symmetric $T^{6} \quad 20$

$\begin{array}{ll}5.1 \text { Generic and special vacua } & 21\end{array}$

5.2 Searching for $g_{\mathrm{s}}$ - emergence of multiple schemata 22

$\begin{array}{ll}5.3 & \text { Minimizing } \mathcal{W}_{0}\end{array}$

5.4 Fixing mass scales 28

6 Conclusions 31

\section{Introduction}

Since the emergence of the string theory landscape [1-3], its predictions (and even the existence of phenomenologically viable vacua) have remained a matter of debate [4-6]. Over the years, several approaches including systematic scans of explicit constructions and statistical analyses have been considered for exploring the landscape [7-21]. The heart of the problem is that the enormous number of vacua in the landscape makes a straightforward scan computationally infeasible. The discrete landscape can be viewed as the complement of a continuum of seemingly consistent low-energy effective field theories (EFTs) that cannot descend from a string compactification, deemed the swampland [22, 23], see [24] for a review. While the latter has received much attention in recent years, progress in data science might allow for systematic studies of the landscape itself, as demonstrated with a variety of techniques such as topological data analysis [25] and machine learning [26-35]. 
In this paper, we study the landscape of string vacua using Genetic Algorithms (GAs) [36-41]. GAs search for optimal solutions to problems with huge input spaces via a natural selection process. The population of potential solutions evolves according to the fitness of individual members. Each individual is specified by its genotype, a string of data that defines the member's location in the input space. An individual's phenotype characterizes its properties as a solution, generally summarized by some fitness function. Via dynamics motivated by natural selection, genetic information related to the fittest members propagates to subsequent generations. Overall, the population adapts to the extrinsic factors implemented by the fitness.

In the past few decades, genetic algorithms have proven useful in diverse areas of physics including particle phenomenology [42-44], astrophysics [45-47] and cosmology [48, 49]. Applications of GAs to the landscape have so far been rather limited [28, 50-55]. This is surprising given that they are very successful in scanning large data sets for viable solutions. Moreover, for deterministic algorithms, difficulties due to the large size of the landscape are exacerbated by computational complexity, as can be seen in several toy models [56-59]. We might hope to avoid some of these issues using stochastic search methods based on natural selection processes, such as GAs. On the deep learning side, a closely related technique is Reinforcement Learning (RL). GAs and RL are both used to train machine agents to solve complex tasks in uncertain environments [60, 61].

Ultimately, we are interested in selecting vacua based on their phenomenological properties such as the cosmological constant or certain desirable low energy spectra and couplings. As a first step, we focus in this paper on three such quantities: the string coupling, the superpotential value in the stabilized vacua, and the moduli masses. Nonetheless, it is straightforward to extend our GA analysis to other contexts. Methods along the lines outlined in this paper can be used e.g. to test the WGC [62] (and its various strong forms [63-68]). As a proof of concept, the problems addressed in this paper are not too computationally intensive, so all the algorithms we used can be implemented in Mathematica. However, it is worth mentioning that there are many publicly available packages for GAs written for different platforms, see e.g. [69-72]. ${ }^{1}$

In this paper, we explore the type IIB flux landscape, for which our genetic algorithms are (mostly) based on the following identifications, see also table 1. The members of a population are distinct string vacua, each determined by a choice of (integer-quantized) fluxes. In other words, the chromosomes are flux vectors of maximal length $4\left(h^{(2,1)}+1\right)$ characterizing the genotype of a vacuum. Solving the F-term constraints for these fluxes determines the VEVs of the various moduli and gives the phenotype of a vacuum. The corresponding fitness is a function of physical observables including masses and couplings. Parent vacua are selected according to their fitness, and breed pairwise to construct child vacua. Parents breed via a crossover procedure where parts of one chromosome are replaced by the corresponding parts of a mate's chromosome. Additionally, mutation modifies some fluxes at random. After solving the F-flatness conditions and checking gauge inequivalence

\footnotetext{
${ }^{1}$ For a maintained list of packages for genetic programming in general, see http://geneticprogramming. com/software/.
} 


\begin{tabular}{|c|c|}
\hline Genetic Algorithms & Landscape \\
\hline individuals & flux vacua \\
\hline chromosome & flux vector \\
\hline alleles & fluxes \\
\hline phenotype & masses, couplings, etc. \\
\hline fitness & function of masses, couplings, etc. \\
\hline boundary conditions & SUSY condition, gauge fixing, tadpole \\
\hline
\end{tabular}

Table 1. Dictionary relating terms used in the context of GAs and the flux landscape.

and the size of tadpoles, we can define a new population by choosing an appropriate number of child vacua as our subsequent generation.

Repeating the above steps for many generations results in a population gathering around a point of maximal fitness, i.e., vacua satisfying physical conditions specified by the fitness. Moreover, we find that GAs are significantly more efficient at finding physical solutions than randomly choosing fluxes. In other words, GAs are inherently superior to brute force approaches. This is a hint that the algorithm exploits some underlying structure of the flux landscape that is unknown at the outset of the search. This learning of structure can be confirmed by studying the "shapes" of individual generations using persistent homology, a technique previously applied to the landscape in [25]. Exploitation of this structure leads to improved efficiency of a GA compared to simulated annealing, see section 4.6 .

GAs, or stochastic search algorithms in general, are not effective for "needle-in-ahaystack" type problems. These problems do not allow for a distinction between incorrect solutions. In other words, all incorrect solutions are equally bad, so the performance of a GA is reduced to that of a random scan. Turning this around, it is important that a GA is searching a fitness landscape that is pseudo-continuous. Stated differently, there should exist a neighborhood around the optimal solution in which the fitness is well-behaved. This can be quantified by considering the fitness-distance correlation $[73,74]$ with distance measured in the input space.

In general, stochastic search algorithms are efficient when the fitness landscape exhibits a funnel-like topography (see [75], and recently in the context of the landscape [76]), so that the optimal solution can be approached via small steps. This encodes itself in a large and negative fitness-distance correlation near the optimal solution, see section 3.3. Strictly speaking, the viability of a given search algorithm is determined empirically. If a solution or near-solution is found, the algorithm may be considered successful. If a solution is not found, either it does not exist, or it is not readily accessible to the algorithm at hand. In the latter case, computing the fitness-distance correlation can demonstrate the lack of a favorable funnel topography. This can sometimes be ameliorated by an alternative choice of problem encoding [77], but in general finding an efficient encoding requires solving the problem itself (in which case no search algorithm is necessary) or finding successful deterministic heuristics, which we aim to avoid due to its problem-specific nature. 
In the presence of a fitness landscape the flow of the population shows a strong pull on certain observables so that the population quickly gathers around suitable solutions. This behavior typically depends on the precise definition of the fitness itself, especially when considering several search parameters. In contrast, "needle-in-a-haystack" situations can only randomly sample the space of solutions since unwanted solutions cannot be distinguished. As we will see, all models considered in this paper do not belong to the class of a "needle-in-a-haystack". It has previously been argued that due to the structure and correlations within the landscape GAs are expected to work well more generally [55]. It seems therefore promising to us that GAs constitute valuable tools to systematically study the landscape.

This paper is organized as follows. First, we review the construction of flux vacua in type IIB compactifications in section 2. We then collect the basic principles of genetic algorithms in section 3. Subsequently, we define an algorithm suitable for studying type IIB flux compactifications. Afterwards, we apply our algorithm to two examples: a hypersurface in the weighted projective space $\mathbf{W P}_{1,1,1,1,4}^{4}$ (section 4 ) and the symmetric $T^{6}$ (section 5). We conclude in section 6.

\section{Flux compactifications}

In this section, we briefly review type IIB flux compactifications on Calabi-Yau orientifolds in the presence of background fluxes, see $[78,79]$ for reviews. In these setups, 3 -form fluxes stabilize the axio-dilaton and complex structure moduli. Due to flux quantization and tadpole cancellation, the resulting space of vacuum solutions is a discretuum distributed over the moduli space $[1,80]$.

We follow the conventions of [81]. Consider a Calabi-Yau threefold $M$ with $h^{(2,1)}$ complex structure moduli. We take a symplectic basis $\left\{A^{a}, B_{b}\right\}$ for the $b_{3}=2 h^{(2,1)}+2$ three-cycles, with $a, b=1, \ldots, h^{(2,1)}+1$. The dual cohomology elements $\alpha_{a}, \beta^{b}$ satisfy

$$
\int_{A^{a}} \alpha_{b}=\delta_{b}^{a}, \quad \int_{B_{b}} \beta^{a}=-\delta_{b}^{a}, \quad \int_{M} \alpha_{a} \wedge \beta^{b}=\delta_{a}^{b} .
$$

From the unique holomorphic three-form $\Omega$, we define the periods $z^{a} \equiv \int_{A^{a}} \Omega, \mathcal{G}_{b} \equiv \int_{B_{b}} \Omega$, which form the $b_{3}$-vector $\Pi(z) \equiv\left(\mathcal{G}_{b}, z^{a}\right)$. Additionally

$$
\int_{M} \Omega \wedge \bar{\Omega}=\bar{z}^{a} \mathcal{G}_{a}-z^{a} \overline{\mathcal{G}}_{a}=-\Pi^{\dagger} \cdot \Sigma \cdot \Pi
$$

in terms of the symplectic matrix

$$
\Sigma=\left(\begin{array}{cc}
0 & 1 \\
-1 & 0
\end{array}\right)
$$

whose entries are $\left(h^{(2,1)}+1\right) \times\left(h^{(2,1)}+1\right)$ matrices. The RR and NSNS 3-form fluxes are quantized and can be expanded in the $\alpha, \beta$ basis as

$$
F_{3}=-(2 \pi)^{2} \alpha^{\prime}\left(f_{a} \alpha_{a}+f_{a+h^{(2,1)}+1} \beta^{a}\right), \quad H_{3}=-(2 \pi)^{2} \alpha^{\prime}\left(h_{a} \alpha_{a}+h_{a+h^{(2,1)}+1} \beta^{a}\right) .
$$


Here, $f$ and $h$ are two integer-valued $b_{3}$-vectors. From now on we set $(2 \pi)^{2} \alpha^{\prime}=1$ and define

$$
\mathbf{N}=\left(f_{1}, \ldots, f_{2 h^{(2,1)}+2}, h_{1}, \ldots, h_{2 h^{(2,1)}+2}\right)^{T}
$$

for later purposes. The fluxes induce a superpotential for the complex structure moduli and axio-dilaton $\phi \equiv C_{0}+\mathrm{i} e^{-\varphi}$, given by [82]

$$
\mathcal{W}=\int_{M} G_{3} \wedge \Omega(z)=(f-\phi h) \cdot \Pi(z) .
$$

In type IIB supergravity the 3-form fluxes only appear in the combination $G_{3} \equiv F_{3}-\phi H_{3}$.

The tree-level $\mathcal{N}=1 \mathrm{~F}$-term scalar potential induced by 3 -form fluxes is of no-scale type, given by

$$
V=\mathrm{e}^{\mathcal{K}}\left(\mathcal{K}^{a \bar{b}} D_{a} \mathcal{W} D_{\bar{b}} \overline{\mathcal{W}}+\mathcal{K}^{\phi \bar{\phi}} D_{\phi} \mathcal{W} D_{\bar{\phi}} \overline{\mathcal{W}}\right)
$$

Here, $\mathcal{K}^{a \bar{b}}$ is the inverse Kähler metric on complex structure moduli space and $D_{a} \mathcal{W}=$ $\left(\partial_{z_{a}}+\left(\partial_{z_{a}} \mathcal{K}\right)\right) \mathcal{W}$ the associated Kähler derivative (similarly for $\left.\phi\right)$. The corresponding mass matrix for the real scalar fields is given by

$$
M_{I J}=\partial_{I} \partial_{J} V
$$

evaluated at a minimum of $V$ with $I, J \in\left\{\operatorname{Re}\left(z_{a}\right), \operatorname{Im}\left(z_{a}\right), \operatorname{Re}(\phi), \operatorname{Im}(\phi)\right\}$.

We are interested in vacua with vanishing $\mathrm{F}$-terms

$$
\begin{aligned}
& D_{\phi} \mathcal{W}=\frac{1}{\bar{\phi}-\phi}(f-\bar{\phi} h) \cdot \Pi(z)=0, \\
& D_{a} \mathcal{W}=(f-\phi h) \cdot\left(\partial_{a} \Pi(z)+\Pi(z) \partial_{a} \mathcal{K}\right)=0
\end{aligned}
$$

The Kähler potential for the axio-dilaton and complex structure moduli is given by

$$
\begin{aligned}
\mathcal{K} & =-\log \left(\mathrm{i} \int_{M} \Omega \wedge \bar{\Omega}\right)-\log (-\mathrm{i}(\phi-\bar{\phi})) \\
& =-\log \left(-\mathrm{i} \Pi^{\dagger} \cdot \Sigma \cdot \Pi\right)-\log (-\mathrm{i}(\phi-\bar{\phi}))
\end{aligned}
$$

The F-flatness conditions (2.9) and (2.10) imply that the $(3,0)$ and $(1,2)$ parts of the fluxes vanish, so that $G_{3}$ is imaginary self-dual, ${ }_{\star_{6}} G_{3}=\mathrm{i} G_{3}$.

The D3-brane charge induced by the fluxes can be written as

$$
N_{\text {flux }}=\int_{M} F_{3} \wedge H_{3}=f \cdot \Sigma \cdot h .
$$

One can show that $N_{\text {flux }}>0$ for imaginary self-dual fluxes. Hence, negative D3-brane charges have to appear to ensure tadpole cancellation. These negative charges can be induced by orientifolding. In this paper, we will not be explicit about orientifolding. We will rather take $L_{\max }$ as an adjustable parameter and, along the lines of [7, 8, 81], consider solutions with

$$
0<N_{\text {flux }} \leq L_{\max }
$$

with remaining charge cancelled by mobile D3-branes. As shown in [25], $L_{\max }$ sets the scale at which interesting structure in the moduli space distribution appears. 
It is crucial that we fix gauge symmetries relating equivalent vacua when running our GA. Otherwise, we might end up with redundancies in our population of vacua. In our case, the symmetry group is given by $\mathcal{G}=\mathrm{SL}(2, \mathbb{Z})_{\phi} \times \Gamma$ where $\mathrm{SL}(2, \mathbb{Z})_{\phi}$ is the S-duality group from type IIB and $\Gamma$ is the modular group acting on the complex structure moduli space. We choose a gauge-fixing prescription in which each vacuum is mapped to the corresponding fundamental domain where we have to keep track of all fluxes. Throughout, we will consider the evolution of stabilized VEVs for the axio-dilaton and complex structure moduli, and functions thereof.

\section{Genetic algorithms}

In this section, we collect the necessary ingredients to study genetic algorithms in the context of string compactifications, see also $[39,42,55]$ for more pedagogical introductions. We begin with a brief summary of GAs. Afterwards, we turn to applications to flux vacua and describe the algorithm applied in the remainder of this paper. We also highlight novel complications encountered when using GAs to search the landscape, namely that consistency conditions are not necessarily preserved by the breeding process. Finally, we elaborate further on the suitability and performance of GAs in the context of flux compactifications.

\subsection{Generalities}

A GA in the sense of $[36,38]$ can be described as follows. One begins with a population of $p$ randomly chosen individuals, each specified by a string of data referred to as the chromosome. The chromosome describes the defining input parameters of each individual, thereby encoding the individual's genotype. The chromosome consists of alleles whose number and values depend on the model in question. Typically, for alleles taking values in a smaller range, a smaller population size can be chosen, see the discussion in section 3.2. The physical characteristics of a member, also called its phenotype, are obtained by computing functions of the genotype. In a physics context, this typically corresponds to a set of physical observables like masses, couplings, or representations. Now, the algorithm proceeds by repeating the following three steps.

First, the process of selection is introduced by declaring certain individuals to be more competitive for breeding than others. This is typically achieved by collecting pairs where the single members in a pair are referred to as parents. As in nature, specific individuals have physical properties distinguishing them from the typical individual and making them more likely to procreate. This preeminence can be taken into account by assigning to each member a fitness based on the phenotype.

Several ways to define the fitness as well as various selection methods have been discussed in the literature, see e.g. [83-97]. In this paper, we mostly focus on the so-called roulette-wheel selection, but see section 5.4. Here, one normalizes the fitness function to be a probability distribution. Pairs are constructed by choosing individuals according to this probability distribution. Note that this method of parent selection explicitly takes self-reproduction into account. 
Secondly, individuals breed to construct a new population, made up of members called children. This is achieved by splicing the two chromosomes of a single pair together. We typically employ a two-point crossover where the parent chromosomes are both cut at the same two randomly selected positions, swapping the middle sections. Afterwards, we pick one of the two new chromosomes at random as the genotype of a new individual in the descendant population.

Thirdly, and this is the major feature responsible for the efficiency of genetic algorithms, the children's chromosomes are altered by mutation. More specifically, for randomly selected children, a fraction of random alleles, typically about $1 \%$, are modified. This is necessary to prevent the algorithm from stagnation. For example, the population can cluster around a local maximum of the fitness, even though a better global maximum is available. The algorithm might never gain knowledge of the latter without mutation. In this sense, mutation should not be viewed as a tool to increase convergence, but more crucially as an integral part of the algorithm itself. Along these lines, we will compare different mutation rates within a simple example in section 4.2. Taking the population size $p$ to remain constant, ${ }^{2}$ the new population is obtained by taking all children, replacing the least fit individual found after mutation with the fittest individual from the previous population. This last step, called elitist selection, forces the maximum fitness to increase monotonically with each generation.

The above three steps form a generation. We repeat them several times, until a limiting rate of convergence is reached. In general, only a certain fraction of the population can reach the optimal solution, see e.g. the discussion in section 1.2 of [55].

\subsection{Genetic algorithms for flux vacua}

In this section we translate the general notion of GAs into the language of type IIB flux compactifications. Throughout this section we employ a notation where $\langle\mathcal{O}\rangle_{A}$ denotes the vacuum expectation value of some quantity $\mathcal{O}$ computed by solving the F-flatness conditions for the fluxes $\mathbf{N}_{A}$.

A population of size $p$ is a set of flux vacua $V_{A}, A=1, \ldots, p$, obtained by solving the F-term constraints $D_{a} \mathcal{W}=0=D_{\phi} \mathcal{W}, a=1, \ldots, h^{(2,1)}$, and fixing the gauge redundancy. Apart from intrinsic geometric quantities specified by the choice of a compactification space, input parameters are only flux numbers $\mathbf{N}_{A}=\left(N_{A}^{1}, \ldots, N_{A}^{2 b_{3}}\right)^{T}$ (recall eq. (2.5)), i.e.,

$$
V_{A}=V\left(\mathbf{N}_{A}\right)=V\left(N_{A}^{1}, \ldots, N_{A}^{2 b_{3}}\right) .
$$

More specifically, $V_{A}$ encodes all elementary information about the vacuum which corresponds in our case to the VEVs of all moduli fields $\left\langle z_{a}\right\rangle_{A}$ and the axio-dilaton $\langle\phi\rangle_{A}$. Hence, each vacuum $V_{A}$ has physical attributes including

- VEVs $\left\langle z_{a}\right\rangle_{A},\langle\phi\rangle_{A}$

- the value of $N_{\text {flux }}\left(\mathbf{N}_{A}\right)$

- the value of $\mathcal{W}_{0}\left(\mathbf{N}_{A}\right)=\langle\mathcal{W}\rangle_{A}$, the string coupling and the moduli masses.

\footnotetext{
${ }^{2}$ Increasing $p$ dynamically during the evolution comes at the expense of increased computational effort. Moreover, the rate of convergence is not improved as long as we start with a large population, see also section 3.2 .
} 
These characteristics specify the vacuum's location in moduli space, the tadpole as well as various mass scales etc. Initially, we choose a certain number of fluxes at random, whereas fluxes of any descendant population are determined through the pairing process (see below). It is crucial that we restrict the tadpole such that $N_{\text {flux }} \leq L_{\max }$, albeit we do not fix it to a certain value. Otherwise, the population might be driven towards solutions with arbitrarily large tadpole. Restricting the size of our tadpole may present limits to the sorts of vacua accessible to our algorithm. For example, it has been noted that in some examples F-term constraints force vacua at weak coupling, large complex structure, and large volume to have large $N_{\text {flux }}[98]$.

The fitness can be used to find vacua with certain values for the VEVs or functions thereof, such as $\mathcal{W}_{0}$, which we collectively denote $\mathcal{O}^{(i)}$ in this section. For a fixed compactification space, the fitness of some vacuum $V_{A}$ will be a function of the fluxes, $F_{A}=F\left(\mathbf{N}_{A}\right)$. Say we are interested in vacua with values $\mathcal{O}^{(i), *}$ for some observables $\mathcal{O}^{(i)}$. (Throughout this paper, we denote with the superscript ${ }^{*}$ the optimal solution within our GA.) We write the value of the observable $\mathcal{O}^{(i)}$ in the vacuum $V_{A}$ as $\mathcal{O}_{A}^{(i)}=\left\langle\mathcal{O}^{(i)}\right\rangle_{A}$. We define the associated fitness $F_{A}$ of $V_{A}$ as

$$
F_{A}=\frac{1}{\mathcal{N}}\left[\sum_{i} w_{i} f_{i}\left(\mathcal{O}_{A}^{(i)}, \mathcal{O}^{(i), *}, \delta \mathcal{O}^{(i)}\right)+b\right] .
$$

Here, we introduce weights $w_{i}$ and a normalisation factor $\mathcal{N}$ so that $\sum_{A} F_{A}=1$. We typically choose $f_{i}$ to be a Gaussian of width $\delta \mathcal{O}^{(i)}$, but other choices also work well. The offset $b$ is necessary to prevent $F_{A}$ from localizing around a local maximum of one of the $f_{i}$. In general, we have to keep in mind that several of the $\mathcal{O}^{(i)}$ could be correlated. Thus, it also has to be checked that the required values $\mathcal{O}^{(i), *}$ are compatible with each other. Finally, the dynamics of the algorithm is generically highly sensitive to the choice of weights $w_{i}$.

Using the fitness, we generate a set of pairs $\left(V_{A}, V_{B}\right)$ of vacua. We allow the two vacua in a pair to be identical. We typically take some number $P>p$ of random pairs, large enough to guarantee enough gauge-inequivalent solutions after breeding satisfying tadpole constraints. This is a minor setback compared to the general algorithm described in section 3.1. There is typically no way to tell whether a pair leads to a viable solution a priori. Still, random search algorithms face similar problems in this context, while they do not benefit from the crucial interplay of selection, crossover and mutation. Thus, we expect our algorithm to be more efficient than a Monte-Carlo procedure, notwithstanding the aforementioned difficulties.

A child vacuum $v_{C}$ is constructed from a pair $\left(V_{A}, V_{B}\right)$ in the following way. First, we construct a new string of fluxes along the lines of section 3.1. That is, the new genotype $\mathbf{n}_{C}=\left(n_{C}^{1}, \ldots, n_{C}^{2 b_{3}}\right)^{T}$ is given by

$$
\mathbf{n}_{C}=\left(N_{A}^{1}, \ldots, N_{A}^{k_{1}}, N_{B}^{k_{1}+1}, \ldots, N_{B}^{k_{2}}, N_{A}^{k_{2}+1}, \ldots, N_{A}^{2 b_{3}}\right)^{T}
$$

where $k_{1}, k_{2} \in\left\{1, \ldots, 2 b_{3}\right\}, k_{1} \leq k_{2}$, are two randomly chosen integers. Mutations are applied by replacing a certain number of fluxes by some new random value. We introduce 
the mutation rate $q_{\text {mut }}$ and perform a mutation on $n_{\text {mut }}$ flux numbers of a single individual whenever $q<q_{\text {mut }}$ for a randomly chosen number $q \in[0,1]$.

Next, all newly obtained strings of fluxes are plugged into the F-term constraints to judge whether they meet all relevant criteria. More specifically, we have to check the existence of solutions, the size of the flux-induced D3-charge $N_{\text {flux }}$ as well as gauge inequivalence. Finally, the new genotype potentially results in a well-defined vacuum $v_{C}=V\left(\mathbf{n}_{C}\right)$.

A new population is defined as a random choice of $p$ children $v_{C}$. As mentioned above, it is hence mandatory that the previous step leads to more than $p$ inequivalent vacua by initially generating $P>p$ pairs. Having defined the population of the next generation, we can continue by computing the associated fitness of each member and going through the same steps again.

We also have to think about the initial population size. We typically restrict to tadpoles smaller than some flux scale $L_{\max }$ and compute the relevant quantities. Since genetic algorithms are designed to find the global maximum of the fitness function, it is necessary to have access to all the parameter space. This means that every allele should include every possible flux choice. We restrict to fluxes in the interval $\left[-L_{\max }, L_{\max }\right]$ for simplicity. ${ }^{3}$ If we take the population size to satisfy

$$
p \geq\left(2 L_{\max }+1\right) 4\left(h^{(2,1)}+1\right),
$$

then this is most certainly true. This is obviously a very naive estimate, but we expect it to be sufficient for the level of our discussion.

\subsection{Schemata and fitness distance correlation}

We call the space parametrized by the $\mathcal{O}_{i}$ the search space, in contrast to the parameter space of fluxes N. A GA converges in search space, but not necessarily in parameter space. By this we mean that the final population is dominated by members for which $\mathcal{O}_{A}^{(i)}$ is close to the optimal solution. More specifically, for two solutions we find

$$
\left|\mathcal{O}_{A}^{(i)}-\mathcal{O}_{B}^{(i)}\right| \lesssim 2 \delta \mathcal{O}^{(i)}
$$

while the distance in parameter space can be arbitrarily large. (This is because the mapping from fluxes to fitness is not one-to-one.) However, a notion of convergence in parameter space also turns out to be useful. As explained in [55], convergence in parameter space occurs for some variables which form so-called schemata, as introduced by Holland [36]. Although Holland's idea has been criticized over the years, ${ }^{4}$ it gives a nice understanding of the generic evolution within a GA, albeit being incomplete. Schemata are characterized by a prototypical behavior of GAs which is that certain parameters quickly converge towards

\footnotetext{
${ }^{3}$ This is typically sufficient for the examples considered here since there are almost no solutions beyond these limits, cf. the discussion at the end of section 4.3.2 and the second paragraph of section 5.4 in [81].

${ }^{4}$ Based on a more mathematical formulation of GAs, it appears misleading to regard static building blocks as being an intrinsic part of the algorithm's evolution, rather than an artefact of the fitness function. In general, these building blocks change dynamically from one generation to the next, cf. chapters 3 and 10 of [96] for a comprehensive discussion. Nonetheless, we generically observe frozen subsets of fluxes characterizing certain regions in moduli space.
} 
one unifying value. To be more precise, schemata represent the crucial features being favorable characteristics of the optimal solution. This can be interpreted as the fact that a small collection of alleles dominate the fitness. As soon as their values are adjusted correspondingly for the majority of the population, the breeding procedure does not affect them anymore. The formal study of schemata results in the following two conclusions. First, the entire population never quite reaches the optimal solution. This is related to the observation that schemata never touch every single individual, see section 1.2 in [55]. Secondly, mutation plays a crucial role in identifying the best schema. As we will see further below, it forces the algorithm to pick out one schema associated to the "global" maximum of $F$. It is important to note that in our case we may not reach the true global maximum, since we typically restrict to certain corners of flux space.

One string-theoretic example where we might expect the domination of various schema is the toy problem of generating fluxes satisfying the tadpole cancellation condition (2.13). For large flux integers (relative to $L_{\max }$ ), satisfying (2.13) requires cancellations between various flux contributions. The various ways flux contributions can cancel represent different (approximate) schema for a search for tadpole-satisfying configurations.

To study the efficiency of GAs for our problems, we consider the $\ell^{1}$-distance from an individual to the fittest individual of a generation in flux space,

$$
D_{A}=\sum_{k=1}^{4\left(h^{(2,1)}+1\right)}\left|N_{A}^{k}-N_{\mathrm{ref}}^{k}\right| .
$$

Here $\mathbf{N}_{\text {ref }}$ is the flux associated to a generation's fittest individual. Recall that this member is carried over to the next generation due to elitist selection. Thus, $\mathbf{N}_{\text {ref }}$ is only replaced if there appears a member of even greater fitness. In the literature on GAs $[73,74],{ }^{5}$ the utility of a GA for a given problem can be quantified by the fitness distance correlation $(\mathrm{FDC})^{6}$

$$
F D C=\frac{1}{p} \sum_{A=1}^{p} \frac{\left(F_{A}-\bar{F}\right)\left(D_{A}-\bar{D}\right)}{\sigma_{F} \sigma_{D}} .
$$

Here, $\bar{F}$ is the average fitness, $\bar{D}$ is the average distance, and $\sigma_{F}, \sigma_{D}$ are the corresponding standard deviations. One infers that models with

- $0.15 \leq F D C \leq 1$ are GA-hard. This class of models is not tackled well by GAs since the fitness correlates with the distance, i.e., the fitness grows with distance.

- $-0.15 \leq F D C \leq 0.15$ are difficult, that is, there is (almost) no correlation between fitness and distance.

\footnotetext{
${ }^{5}$ See also [55] for a discussion of FDC in a physics context.

${ }^{6}$ Notice however that in the present context establishing the FDC in the original sense seems out of reach. This is because $D_{A}$ is in the literature assumed to be the distance to the nearest global maximum. To compute $D_{A}$ in this case, we would have to know about the position of every solution in advance. If this were the case, we would have started with a deterministic approach right from the beginning. As previously noted, models motivated by string compactifications are most probably NP-hard. We are therefore using a different definition of $D_{A}$ which will allow us to judge whether certain questions are GA-hard or GA-easy.
} 
- $-1 \leq F D C \leq-0.15$ are GA-easy. The anti-correlation of fitness and distance implies that the fitness is maximized as the global optimum is approached. Hence, these tasks are suitable for running a GA.

When using the algorithm described in section 3.2, it can be useful to fix certain parameters, while keeping others dynamical. For instance, the width or support of our fitness function can be chosen to decrease to force the population to adapt more and more to a certain value. This is especially helpful when trying to find vacuum solutions sharing certain properties at a given level of accuracy.

To summarize, we can expect the initial population to quickly approach the optimal solution due to the emergence of schemata. Subsequently, there is period of minor adjustments of the remaining flux parameters.

\section{Calabi-Yau hypersurface}

In this section, we first review the definition of the hypersurface in question. Next, we explain the general characteristics of our GA. We compare the results to GAs with different breeding mechanisms and to a Metropolis algorithm. We perform only a single search here before we come to more interesting applications in the upcoming section.

\subsection{Expansion around the conifold locus}

In this section we investigate a Calabi-Yau threefold arising as a hypersurface in the weighted projective space $\mathbf{W} \mathbf{P}_{1,1,1,1,4}^{4}$, defined by

$$
\sum_{i=1}^{4} x_{i}^{8}+4 x_{0}^{2}-8 \psi x_{0} x_{1} x_{2} x_{3} x_{4}=0 .
$$

Its Hodge numbers are given by $h^{(1,1)}=1$ and $h^{(2,1)}=149$. We focus on the orientifold taking $x_{0} \rightarrow-x_{0}, \psi \rightarrow-\psi$ along with worldsheet parity reversal, which arises from $\mathrm{F}$ theory compactified on a Calabi-Yau fourfold defined as a hypersurface in $\mathbf{W P}_{1,1,1,1,8,12}^{5}$. This amounts to tadpole with $L_{\max }=972$ [99]. As described in [81, 99, 100], (4.1) has a discrete symmetry group $\Gamma=\mathbb{Z}_{8}^{2} \times \mathbb{Z}_{2}$, and any complex structure deformation except the $\psi$-term is charged under $\Gamma$. By working in a regime where only fluxes consistent with $\Gamma$ are turned on, we can neglect these charged moduli and consistently solve for the periods for the axio-dilaton $\phi$ and uncharged modulus $\psi$.

We are particularly interested in flux vacua near the conifold point $\psi=1$, for which the periods can be written in terms of $x=1-\psi,|x| \ll 1$, as [100]

$$
\begin{aligned}
\mathcal{G}_{1}(x) & =(2 \pi \mathrm{i})^{3}\left[a_{0}+a_{1} x+\mathcal{O}\left(x^{2}\right)\right], \\
\mathcal{G}_{2}(x) & =\frac{z^{2}(x)}{2 \pi \mathrm{i}} \ln (x)+(2 \pi \mathrm{i})^{3}\left[b_{0}+b_{1} x+\mathcal{O}\left(x^{2}\right)\right], \\
z^{1}(x) & =(2 \pi \mathrm{i})^{3}\left[c_{0}+c_{1} x+\mathcal{O}\left(x^{2}\right)\right], \\
z^{2}(x) & =(2 \pi \mathrm{i})^{3}\left[d_{0}+d_{1} x+\mathcal{O}\left(x^{2}\right)\right] .
\end{aligned}
$$


Here, the constants $b_{0}, d_{0} \in \mathbb{R}, a_{0}, a_{1}, d_{1} \in \mathbb{i} \mathbb{R}$ and $b_{1}, c_{0}, c_{1} \in \mathbb{C}$ can be found in section 5.1 of [100]. To first order in $x \equiv 1-\psi$, the F-flatness conditions result in

$$
\begin{gathered}
\phi=\frac{f_{1} \bar{a}_{0}+f_{2} \bar{b}_{0}+f_{3} \bar{c}_{0}}{h_{1} \bar{a}_{0}+h_{2} \bar{b}_{0}+h_{3} \bar{c}_{0}}+\mathcal{O}(|x| \ln |x|) \\
\ln (x)=-\frac{2 \pi i}{d_{1}}\left[\frac{\left(f_{1}-\phi h_{1}\right)\left(a_{1}-\frac{\mu_{1}}{\mu_{0}} a_{0}\right)+\left(f_{2}-\phi h_{2}\right)\left(b_{1}-\frac{\mu_{1}}{\mu_{0}} b_{0}\right)}{f_{2}-\phi h_{2}}+\right. \\
\left.\frac{\left(f_{3}-\phi h_{3}\right)\left(c_{1}-\frac{\mu_{1}}{\mu_{0}} c_{0}\right)+\left(f_{4}-\phi h_{4}\right) d_{1}}{f_{2}-\phi h_{2}}\right]-1
\end{gathered}
$$

Using Monte Carlo simulations, the authors of [100] explicitly showed that vacua cluster near the conifold point, confirming the expectation from the continuous flux approximation of [7].

In the remainder of this section, we perform all algorithms with

$$
L_{\max }=972, \quad L=100 .
$$

Since we work in the conifold regime, the VEV of the complex structure modulus $x$ is typically driven towards tiny values. This is because the flux vacua cluster around the conifold point.

\subsection{Analyzing different mutation rates}

Let us now find flux vacua with a certain value of the superpotential $\mathcal{W}_{0}$. This can be relevant when, e.g., looking for vacua that are applicable for any of the three branches of Kähler moduli stabilisation, namely KKLT [101], LVS [102] or Kähler uplifting [103], which generically require $\left|\mathcal{W}_{0}\right| \ll 1,\left|\mathcal{W}_{0}\right| \gtrsim 1$ and $\left|\mathcal{W}_{0}\right| \sim \mathcal{O}(1 \ldots 10)$ respectively. For simplicity, we only search for a specific absolute value of $\left|\mathcal{W}_{0}\right|$, although fixing real and imaginary separately is also within the capabilities of our GA. In the following, we use $\mathcal{W}_{0}$ to denote the absolute value of the superpotential. We run the algorithm with the parameters

$$
\mathcal{W}_{0}^{*}=50000, \quad \delta \mathcal{W}=2000, \quad p=1000, \quad N_{\text {gen }}=50 .
$$

Here, we choose not to apply any crossover, but restrict to the effects of selection and mutation. As we will see in the remainder of this paper, this is already sufficient to find (presumably local) fitness maxima. We discuss the impact of crossover on these results in section 4.4. To guarantee the optimal outcome for a GA, it is necessary to adjust the number of mutations as well as the mutation rate. Here, we run our algorithm several times with $n_{\text {mut }}$ mutations per mutated child and mutation rate $q_{\text {mut }}$.

In order to find the optimal mutation scheme, we compare the convergence around a fixed neighborhood around $\mathcal{W}_{0}^{*}$, cf. figure 1. First of all, we observe that applying two mutations per child (purple line) results in an insufficient convergence rate. This behavior is no surprise because, having only 8 independent fluxes to begin with, two mutations overshadow the underlying structure of the optimal solution. In contrast, one mutation results in a seemingly stable evolution over all generations with high convergence rate. 


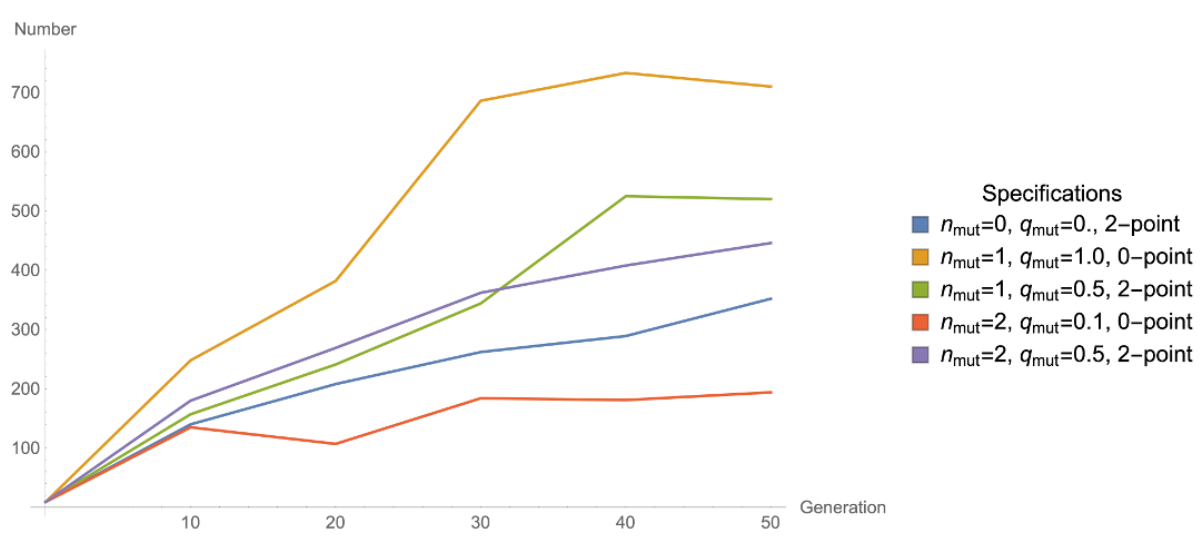

Figure 1. Number of members in neighborhoods of radius $r=500$ around $\mathcal{W}_{0}^{*}$ for zero, one or two mutations and different mutation rates.

Moreover, by comparing the yellow and green line in figure 1 we deduce that the search for $\mathcal{W}_{0}$ considered in this section does not seem to strongly depend on $q_{\text {mut }}$. Nonetheless, we emphasize that it is crucial to scan over different mutation rates in all examples discussed below. Otherwise, the GA's ability to find certain schemata associated to the optimal solution is undermined.

All in all, we conclude that mutation really is an essential ingredient for the success of our algorithm. For our purposes at most one flux mutation per individual seems to be a good guess. However, the mutation rate has to be adjusted accordingly to guarantee the optimal outcome. As we will see below, this typically depends on the specific task.

\subsection{Genetic algorithm dynamics}

After having investigated different mutation rates, we describe the generic features of a GA's evolution. We proceed by running the algorithm using

$$
\mathcal{W}_{0}^{*}=50000, \quad \delta \mathcal{W}=2000, \quad p=1000, \quad N_{\text {gen }}=100, \quad q_{\text {mut }}=0.5 .
$$

In practice, we are interested in solutions that approximately satisfy $\mathcal{W}_{0} \approx \mathcal{W}_{0}^{*}$ up to small deviations. We therefore decrease $\delta \mathcal{W}$ after 50 generations by $50 \%$ every 20 generations. In doing so, we change the fitness landscape and induce an additional force on the flow of the population. As we will see below, this allows us to find about $93 \%$ of the flux vacua localized in the range $\left[\mathcal{W}_{0}^{*}-50, \mathcal{W}_{0}^{*}+50\right]$.

Figure 2 shows the number of individuals in neighborhoods of different sizes $r$ around the optimal solution. During the first 50 generations with $\delta \mathcal{W}=2000$, we observe a sharp incline of the blue and orange curve corresponding to $r=1000$ and $r=750$ respectively. Hence, the population quickly moves towards the optimal solution, but remains mostly outside a neighborhood with $r=500$. After decreasing $\delta \mathcal{W}$, we observe another sharp incline, but this time in the green $(r=500)$ and red $(r=250)$ curve. In the final population about $93 \%$ of individuals have the optimal value of $\mathcal{W}_{0}$ to an accuracy of $0.1 \%$.

We show the distribution of our population in the $g_{\mathrm{s}}-\mathcal{W}_{0}$-plane on the left of figure 3. At the beginning, the population (blue dots) is randomly scattered across random values of $\mathcal{W}_{0}$ and $g_{\mathrm{s}}$. After 40 generations, the distribution of $\mathcal{W}_{0}$ and $g_{\mathrm{s}}$ exhibits a clear structure 


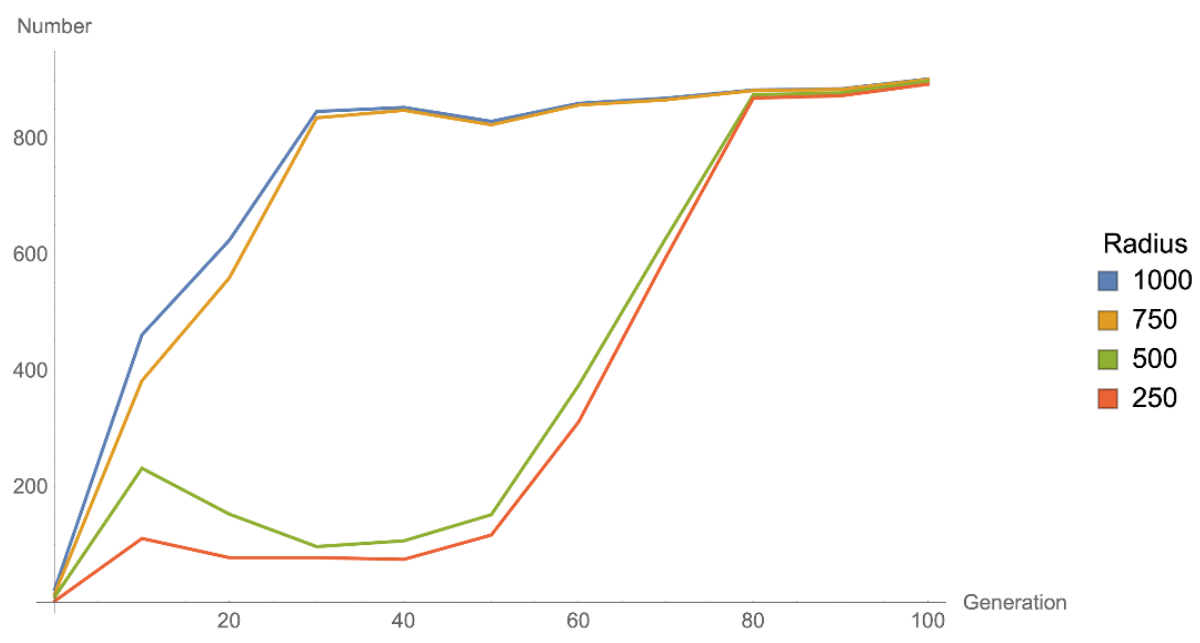

Figure 2. Number of individuals within different ranges around $\mathcal{W}_{0}^{*}$.

in the sense that the individuals around $\mathcal{W}_{0}^{*}$ form almost straight lines in $g_{\mathrm{s}}$-direction. As expected for GAs, a certain fraction of individuals is found far away from the optimal solution. However, the majority of vacua strongly clusters around $\mathcal{W}_{0}^{*}$ which is clearly visible from figure 3. After having decreased $\delta \mathcal{W}$, the distribution of the final population leaves only one of the previous $g_{\mathrm{s}}$-lines present which is closest to $\mathcal{W}_{0}^{*}$. We interpret this behavior as an indication of improved convergence related to the reduction of $\delta \mathcal{W}$. The FDC (3.7) averaged over all generations results in FDC $=-0.53$ which is within the GAeasy regime. This is in good agreement with the above qualitative observations. Thus, we find reason to believe that our definition of an approximate notion of FDC (3.6) is well-suited for our purposes.

Next, let us consider the distribution of $\phi$ in the fundamental domain as depicted on the right hand side of figure 3. Interestingly, after 40 generations the flux vacua mostly align with straight lines pointing towards the origin. These lines seemingly form a symmetric pattern. On top of that, short vertical lines appear at the outer region of the fundamental domain. This pattern survives until the termination of the algorithm and, even more importantly, is enforced during the evolution. We interpret this result as the manifestation of the schemata associated to the optimal value of $\mathcal{W}_{0}$. That is, the chromosomes of individual fluxes share specific properties that result in the observed alignment of $\phi$-VEVs. This interpretation is further supported by comparing different crossover and breeding mechanisms in the next two sections.

To understand these results further, we use persistent homology to analyze the topological structure of the distribution in flux space, applying the methods used in [25, 104], see also [105-110] for more elaborate introductions. Persistent homology has previously been used to characterize distributions of string vacua, and identify loci in moduli space related to phenomenologically interesting properties.

Applied to the scenario under consideration, we compare the flux distributions of populations at different generations during the GA's evolution. We specifically consider the persistence diagrams associated to the data set of fluxes for a single generation. These 

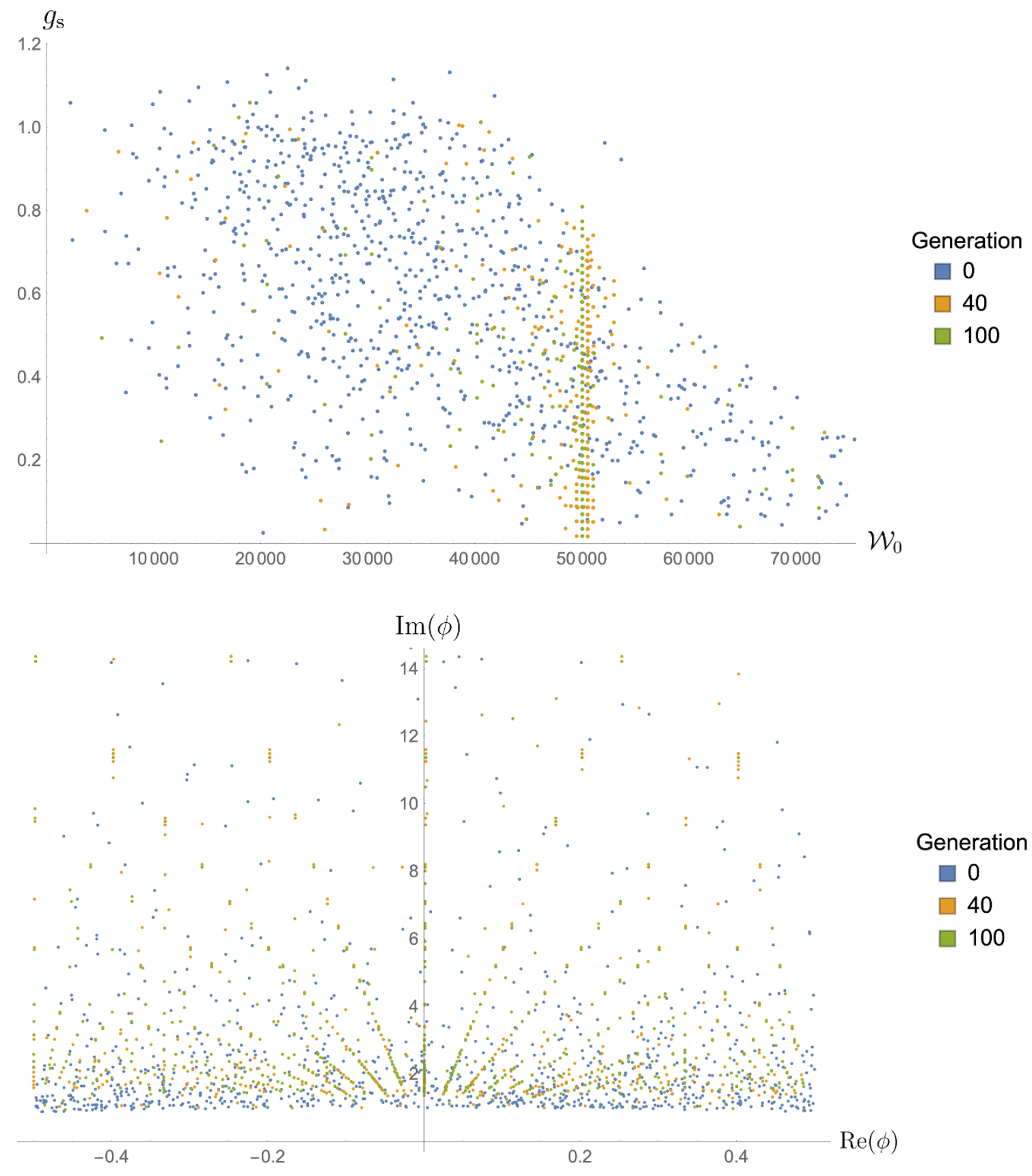

Figure 3. Distribution of $g_{\mathrm{s}}, \mathcal{W}_{0}$ and $\phi$ for three generations.

diagrams encode the two scales at which certain higher-dimensional topological features in the set of fluxes first emerge and vanish. In figure 4, we compare the flux configurations of the initial (top) and the final (bottom) population. ${ }^{7}$ The plot on the top shows a lot

\footnotetext{
${ }^{7}$ More specifically, we consider the distribution of constrained fluxes, cf. section 4.5 for details. These fluxes are eventually fixed by evolution of the GA. Restricting to the constrained fluxes allows one to observe the emergence of schemata during the GA's evolution.
} 

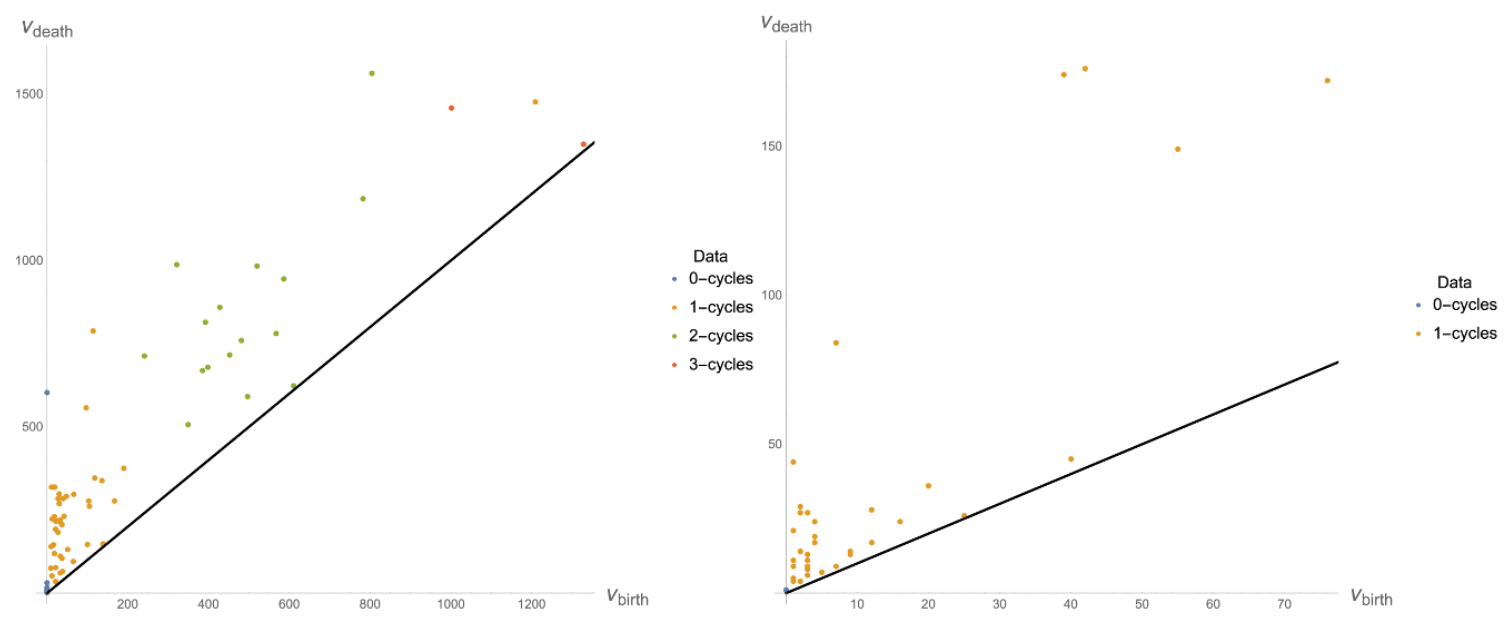

Figure 4. Persistence diagram for the population in the initial (left) and final (right) generation in flux space.

of structure in terms of the presence of various $k$-cycles, albeit most die very quickly. Randomly choosing the initial fluxes results in regions of non-trivial topology. In contrast, the distribution of the final population on the bottom of figure 4 does not show much topologically interesting structure. Moreover, the majority of observed features are very short-lived in terms of the associated scales. This indicates that (most) fluxes form a cluster which does not exhibit any non-trivial topological properties at large scales.

\subsection{The role of crossover}

The takeaway message of the previous section is that a GA can be applied even without performing any crossover. The true power of GAs typically comes only to light when having a dynamical interaction of selection, crossover and mutation. In the remainder of this section, we would like to contrast different choices for running the GA. For instance, one can consider various crossover operators at the same time to increase the GA's performance. This is necessary since different tasks generally require the application of suitable crossover procedures. Here, we restrict to a 2-point crossover operator as explained in section 3.2.

Using the same initial population as previously, we find the evolution depicted as the orange line in figure 5. If we compare the blue (no crossover) and orange (2-point crossover) line, we observe that applying a non-trivial crossover operator leads to a more stable evolution, especially at early stages. Recall that we reduce the support of the fitness at generation 50. At this point of the GA's evolution without crossover the population seemingly feels a strong pull towards the optimal solution. In contrast, the convergence rate with 2-point crossover increases almost monotonically. Thus, although the final population is qualitatively the same, we find significant difference in the overall evolution.

The previously mentioned structures in figure 3 emerge with 2-point crossover as well which is a strong indication for a characteristic feature associated to the optimal solution, see the discussion in the upcoming section. We also analyzed the resulting distribution of 


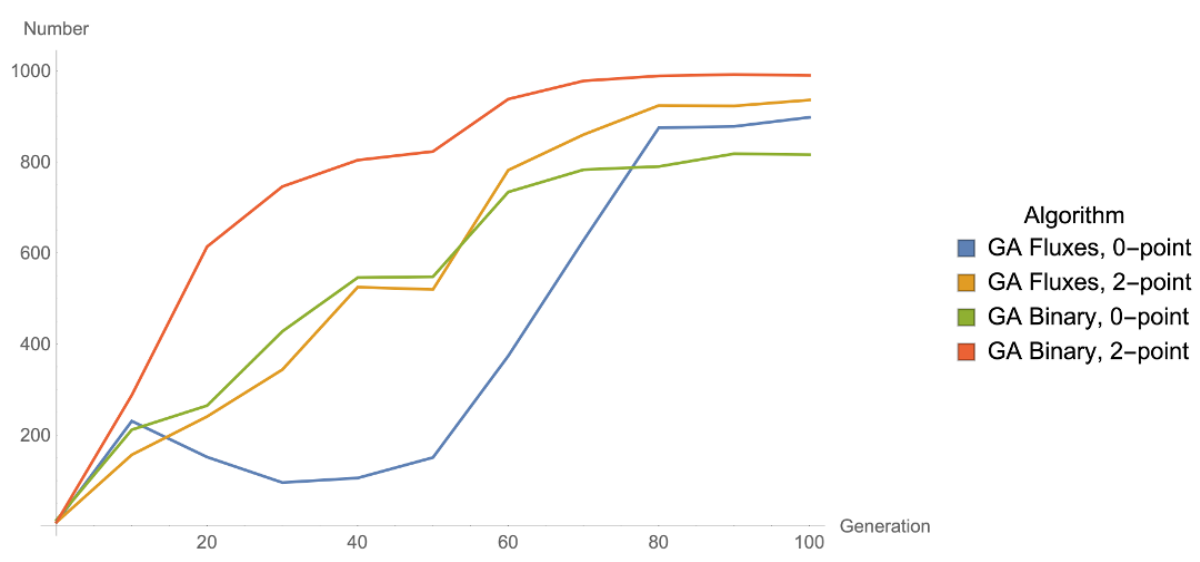

Figure 5. The number of individuals within a range of 500 around $\mathcal{W}_{0}^{*}$ for the algorithms of sections $4.3,4.4$ and 4.5 .

constrained fluxes in the spirit of the previous section. Indeed, the persistence diagram for the final population mirrors the results of figure 4 .

\subsection{Comparing different breeding mechanisms}

There is another way of implementing a genetic algorithm which might be useful when large input parameters appear. Recall that, in order to guarantee that the algorithm can access most of the landscape, we have to ensure that the whole range of alleles appears within each individual locus of a chromosome. This means that the necessary population size scales with the maximally allowed allele. Hence, it might be useful to interpret each flux as a single chromosome by encoding it in a binary form. As opposed to the breeding and crossover mechanism explained in section 3.2, we now perform crossovers for each individual flux. That is, we act on 8 strings of zeros and ones. It is expected that the necessary population size is drastically reduced in this way. In fact, one finds that for a chromosome of length $\ell$ [55]

$$
p_{\min } \approx 1+\frac{1}{\log (2)} \log \left(\frac{-\ell}{\log \left(P_{*}\right)}\right)
$$

with $P_{*}$ being the probability with which every allele should appear at each locus at least once. This is generally much smaller than the minimum population size for alleles taking values in $\left[-L_{\max }, L_{\max }\right]$.

In this section, we compare the performance of both methods, applying 0- and 2-point crossover for the same population size $p=1000 .^{8}$ First, the overall evolution is very alike to the distribution shown in figure 3. It is worth mentioning that the observed pattern in the distribution of $\phi$-VEVs also emerges here independently of whether we perform 0- or 2-point crossover. Thus, we are convinced that this linear structure is not related to the applied crossover procedure, but rather a property of the vacua close to the optimal solution.

\footnotetext{
${ }^{8}$ The precise value of a minimal population size does not matter in our simple models. However, for applications with more degrees of freedom the advantage of a reduced minimal $p$ from binary encoding can be substantial. For our purposes, this way of encoding crossovers gives similar results.
} 
In figure 5, we see that there are only minor differences in the overall performance. Hence, this way of performing crossovers and mutations might be advantageous in higherdimensional models than considered here. The point is that solving the equations of motion in high-dimensional spaces is computationally very costly. Thus, working with smaller population sizes provides a welcome reduction in computational expense.

As before, we observe a strong clustering also for the case of binary crossover. This becomes apparent by performing a Principal Component Analysis (PCA) on the final population. In general, PCA is useful for determining sets of linearly uncorrelated variables. Applied to the flux distribution of our population we gain information about the effective dimensionality of the resulting (discrete) hypersurface. That is, some fluxes will take specific values as we argued previously in the context of schemata in section 3.3, while others remain arbitrarily distributed over some range of flux values $[-L, L]$. PCA organizes the full flux space into orthogonal dimensions of decreasing variance. The intuition is that most data actually lives on a hyperplane of smaller dimension than the naive dimensionality of the data set. This can be quantified by examining the percentage of the data's variance that is retained when the data is projected onto a particular hyperplane.

One subtlety is that our gauge-fixing condition forces different flux components to take values in ranges of different scales. Naive application of PCA would then artificially inflate the importance of fluxes taking values in a large range. To remove this artifact, we first rescale the flux components in the initial population so that each component has zero mean and unit variance. The same scaling transformation is then applied to the final transformation.

For the final population obtained in section 4.3 and section 4.4, we find that the fluxes live on an effectively 1-dimensional hypersurface, with $92.84 \%$ (no crossover) and $89.57 \%$ (2-point crossover) of the variance in the first component. Similarly, binary encoding leads to significantly more clustering with $99.63 \%$ (no crossover) and 92.96\% (2-point crossover) variance in the first component. Qualitatively, both crossover procedures give rise to a very similar evolution of the variance.

\subsection{Comparison to a Metropolis algorithm}

In this section, we compare the results of our genetic algorithm to those of random walk approaches. More specifically, we consider a Metropolis algorithm [111] or rather its related variant of simulated annealing [112]. As before, we start with a randomly sampled generation of flux vacua. In contrast to the GA, we take a random step from each point in flux space until we find a new solution to the F-term equations with small enough tadpole. Explicitly, if $\mathbf{N} \in \mathbb{Z}^{8}$ is some chromosome in our population, then a new flux $\mathbf{M} \in \mathbb{Z}^{8}$ is chosen as

$$
\mathbf{M}=\mathbf{N}+r \mathbf{q}
$$

where $\mathbf{q} \in \mathbb{Z}^{8},\left|q_{i}\right| \leq 1$, is a random vector and $r \in \mathbb{N}$ some fixed step size. The vector $\mathbf{q}$ must be chosen such that $\mathbf{M}$ also leads to a solution of the F-term constraints with $N_{\text {flux }}(\mathbf{M}) \leq L_{\max }$. Practically, this means that we have to perform many such steps and test the criteria for a viable physical flux choice. 


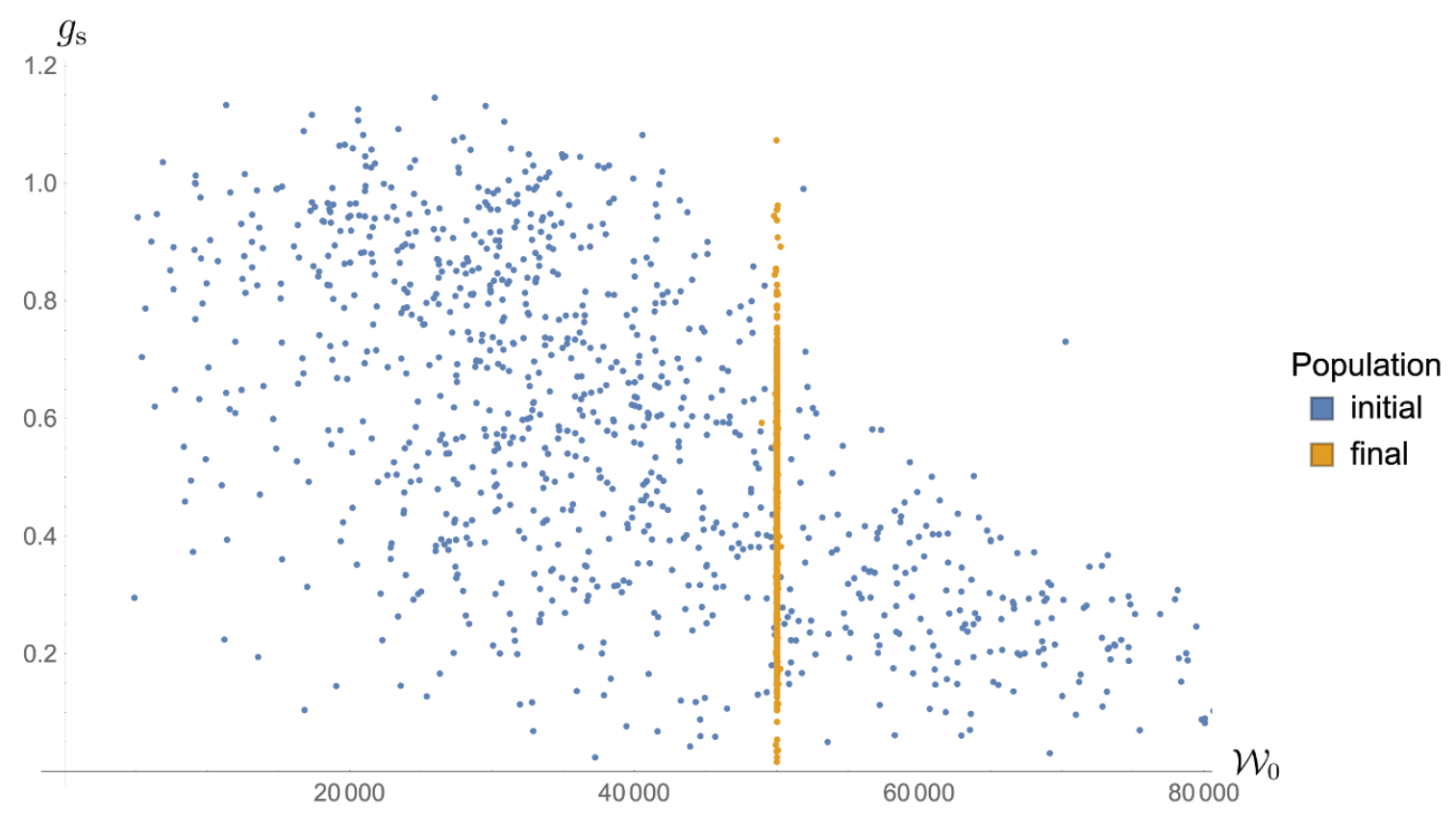

Figure 6. The distribution for $g_{\mathrm{s}}$ and $\mathcal{W}_{0}$ obtained from a Metropolis algorithm.

In order to define a new population, we evaluate an energy functional $E$ given by the difference in fitness

$$
E=F(\mathbf{N})-F(\mathbf{M})
$$

If $E \leq 0$, the new configuration $\mathbf{M}$ is energetically favorable. In this case, $\mathbf{M}$ is carried over to the new population. If $E>0$, we define a probability

$$
P_{T}(E)=\mathrm{e}^{-E / T}
$$

with some "temperature" $T$ and draw a random number $q \in[0,1]$. If $q<P_{T}(E)$, $\mathbf{M}$ is chosen as a member of the next generation.

Again, we use the same initial flux choices and let the algorithm run for 1000 steps for each individual. We choose $r=1$ and determine the initial temperature $T$ for each vacuum solution individually. That is, we fix an initial acceptance rate of about $20 \%$ by considering 100 random steps $\mathbf{q}$ before starting the evolution. We decrease the temperature by a factor of 10 every 100 steps. In total, we find that $96 \%$ of individuals can be found within the range $\left[\mathcal{W}_{0}^{*}-50, \mathcal{W}_{0}^{*}+50\right]$. If we compare the overall evolution of the distribution of $g_{\mathrm{s}}$ and $\mathcal{W}_{0}$ in figure 6 with the one for the GA in figure 3, it becomes clear that this method, with each individual walking independently of the others, does not exploit correlations between different vacua. Although the population gets close to the optimal solution, it shows less structure than the equivalent plots in figure 3. Similarly, we checked that the distribution of $\phi$-VEVs does not exhibit the symmetric pattern of figure 3. Qualitatively, this can be understood as follows. After some generations within a GA, all vacua are in a sense related to each other due to the applied selection process. On the contrary, the random walk 


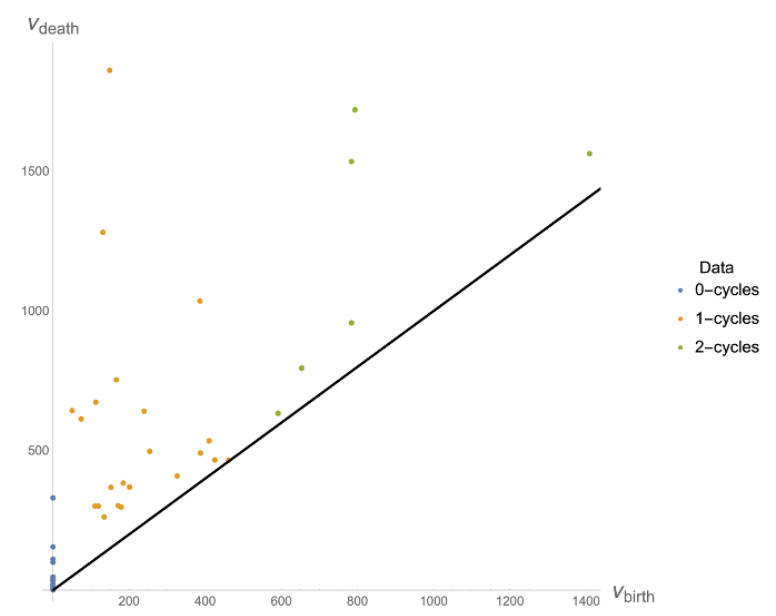

Figure 7. The persistence diagram for the final population obtained with the Metropolis algorithm. There is significantly more large scale structure to be observed than in the analogous plot on the bottom of figure 4 .

approach does not link solutions in a similar fashion. Thus, there is no reason for them to share characteristics, albeit the population gathers around the optimal value $\mathcal{W}_{0}^{*}$.

Since the final population is randomly distributed around the optimal solution, there should be less clustering in flux space. By this reasoning, we should expect the distribution of fluxes in the final distribution to have a much richer structure than for a GA. This can be seen by comparing the persistence diagrams. Recall that in figure 4 we observed that the final population resembles a cluster in flux space. In contrast, the persistence diagram of the final population associated to the Metropolis algorithm in figure 7 exhibits more long-lived higher-dimensional features. That is, there are a greater number of long-lived 1and 2-cycles.

As before, we performed PCA on the flux distribution. Here, the variance in the first component increases to only $54.6 \%$ in contrast to $\gtrsim 89 \%$ for the previous algorithms. That is, the final population obtained via the Metropolis algorithm is less constrained in flux space. This is to be expected from dynamics of the algorithm and already confirmed via TDA.

Finally, we note that in our experiments, Metropolis was less efficient than GAs in two senses. First, the random steps taken by Metropolis were less efficient in finding physical vacua than our GA. As previously argued, crossover should be more efficient in finding physical vacua than taking random steps. Second, even in terms of physical steps, Metropolis took longer than our GAs. We found that it took Metropolis an average of 336 physical steps to match the performance of a GA after 80 generations.

\section{$5 \quad$ Symmetric $T^{6}$}

In this section we consider compactifications on a symmetric $T^{6}$. One interesting feature of the symmetric $T^{6}$ is the existence of vacua with vanishing tree-level superpotential. These special vacua give rise to the opportunity to study their emergence within GAs. A major issue with finding such vacua is that they are quite scarce in comparison to generic vacua. 
Hence, we cannot take for granted that GAs can identify these exceptional cases within the landscape.

\subsection{Generic and special vacua}

We follow the conventions of [81]. The symmetric torus can be viewed as a direct product of three $T^{2}$ setting the modular parameters $\tau \equiv \tau_{1}=\tau_{2}=\tau_{3}$ all equal. The moduli space has two complex dimensions, so we have upon gauge fixing 8 independent flux parameters. Let us first parametrize a general $T^{6}$ before we specialize to the symmetric case further below. We define coordinates $x^{i}, y^{i}$ for $i=1,2,3$ with periodicity $x^{i} \equiv x^{i}+1, y^{i} \equiv y^{i}+1$ such that the three holomorphic 1-forms can be written as $\mathrm{d} z^{i}=\mathrm{d} x^{i}+\tau^{i j} \mathrm{~d} y^{j}$. We take the orientation

$$
\int \mathrm{d} x^{1} \wedge \mathrm{d} x^{2} \wedge \mathrm{d} x^{3} \wedge \mathrm{d} y^{1} \wedge \mathrm{d} y^{2} \wedge \mathrm{d} y^{3}=1
$$

and choose a symplectic basis for $H^{3}\left(T^{6}, \mathbb{Z}\right)$, namely

$$
\begin{aligned}
\alpha^{0} & =\mathrm{d} x^{1} \wedge \mathrm{d} x^{2} \wedge \mathrm{d} x^{3}, & & \alpha_{i j}=\frac{1}{2} \epsilon_{i l m} \mathrm{~d} x^{l} \wedge \mathrm{d} x^{m} \wedge \mathrm{d} y^{j}, \\
\beta^{i j} & =-\frac{1}{2} \epsilon_{j l m} \mathrm{~d} y^{l} \wedge \mathrm{d} y^{m} \wedge \mathrm{d} x^{i}, & \beta^{0} & =\mathrm{d} y^{1} \wedge \mathrm{d} y^{2} \wedge \mathrm{d} y^{3} .
\end{aligned}
$$

The holomorphic 3-form can be written as

$$
\Omega=\mathrm{d} z^{1} \wedge \mathrm{d} z^{2} \wedge \mathrm{d} z^{3} .
$$

We can expand the 3 -form fluxes in terms of the symplectic basis (5.2)

$$
\begin{aligned}
& F_{3}=a^{0} \alpha^{0}+a^{i j} \alpha_{i j}+b_{i j} \beta^{i j}+b_{0} \beta^{0} \\
& H_{3}=c^{0} \alpha^{0}+c^{i j} \alpha_{i j}+d_{i j} \beta^{i j}+d_{0} \beta^{0} .
\end{aligned}
$$

For a symmetric $T^{6}$, we take

$$
\tau^{i j}=\tau \delta^{i j}
$$

This is equivalent to taking the $T^{6}$ to be factorizable as three two-tori with equal modular parameter. Similarly, the fluxes get reduced to

$$
a^{i j}=a \delta^{i j}, \quad b_{i j}=b \delta_{i j}, \quad c^{i j}=c \delta^{i j}, \quad d_{i j}=d \delta_{i j} .
$$

The superpotential takes the simple form

$$
\mathcal{W}=P_{1}(\tau)-\phi P_{2}(\tau)
$$

where $P_{i}$ are cubic polynomials in $\tau$, i.e.,

$$
\begin{aligned}
& P_{1}(\tau)=a^{0} \tau^{3}-3 a \tau^{2}-3 b \tau-b_{0}, \\
& P_{2}(\tau)=c^{0} \tau^{3}-3 c \tau^{2}-3 d \tau-d_{0} .
\end{aligned}
$$

The Kähler potential for $\tau$ and $\phi$ reads

$$
\mathcal{K}=-3 \log (-i(\tau-\bar{\tau}))-\log (-i(\phi-\bar{\phi}))
$$

and the D3-brane charge induced by fluxes corresponds to

$$
N_{\text {flux }}=b_{0} c^{0}-a^{0} d_{0}+3(b c-a d) .
$$


The F-term constraints can be written in the form

$$
\begin{aligned}
& P_{1}(\tau)-\bar{\phi} P_{2}(\tau)=0 \\
& P_{1}(\tau)-\phi P_{2}(\tau)=(\tau-\bar{\tau})\left(P_{1}^{\prime}(\tau)-\phi P_{2}^{\prime}(\tau)\right)
\end{aligned}
$$

For non-zero VEV of the superpotential $\mathcal{W}_{0} \neq 0$, the axio-dilaton can be obtained using (5.12) so that

$$
\phi=\frac{\overline{P_{1}(\tau)}}{\overline{P_{2}(\tau)}} .
$$

Plugged into (5.13), one finds for $\tau=x+i y$

$$
\begin{aligned}
& q_{1}(x) y^{2}=q_{3}(x), \\
& q_{0}(x) y^{4}=q_{4}(x) .
\end{aligned}
$$

The $q_{i}$ are polynomials in $x$ which have for instance been computed in the appendix of [81]. Surprisingly, multiplying both (5.15) and (5.16) to eliminate $y$, a cubic (rather than sextic) equation in $x$ remains so that $x$ can be found by solving

$$
\alpha_{3} x^{3}+\alpha_{2} x^{2}+\alpha_{1} x+\alpha_{0}=0 .
$$

The coefficients $\alpha_{i}$ are combinations of flux integers and can again be found in [81].

Solutions with $\mathcal{W}_{0}=0$ satisfy

$$
P_{1}(\tau)=P_{2}(\tau)=0
$$

Thus, the solution for $\phi$ simply reads

$$
\phi=\frac{P_{1}^{\prime}(\tau)}{P_{2}^{\prime}(\tau)} .
$$

As shown in [113], these solutions obey the special property that $P_{1}$ and $P_{2}$ must factorize over the integers, cf. section 4.3.3 in [81]. Recently, it was shown in [25] using persistent homology that the solutions with $\mathcal{W}_{0}=0$, when combined with flux quantization and tadpole cancellation, exhibit a different structure in the moduli space than generic vacua. We will see in section 5.3 that this also plays an important role when applying GAs to minimizing $\mathcal{W}_{0}$.

\subsection{Searching for $g_{\mathrm{s}}$ - emergence of multiple schemata}

In this section, we discuss the emergence of various correlations and schemata within the GA's evolution. For the sake of simplicity, let us first look for solutions with a certain value of $g_{\mathrm{s}}$. We begin with the following choice of parameters

$$
g_{\mathrm{s}}^{*}=0.3, \quad \delta g_{\mathrm{s}}=0.05, \quad p=10000, \quad N_{\mathrm{gen}}=100, \quad L_{\max }=500, \quad q_{\mathrm{mut}}=1 .
$$

We use a Gaussian to define the fitness, but e.g. a combination of Heaviside-functions works equally well. After half of the generations, we divide $\delta g_{\mathrm{s}}$ by a factor of 2 every 10 


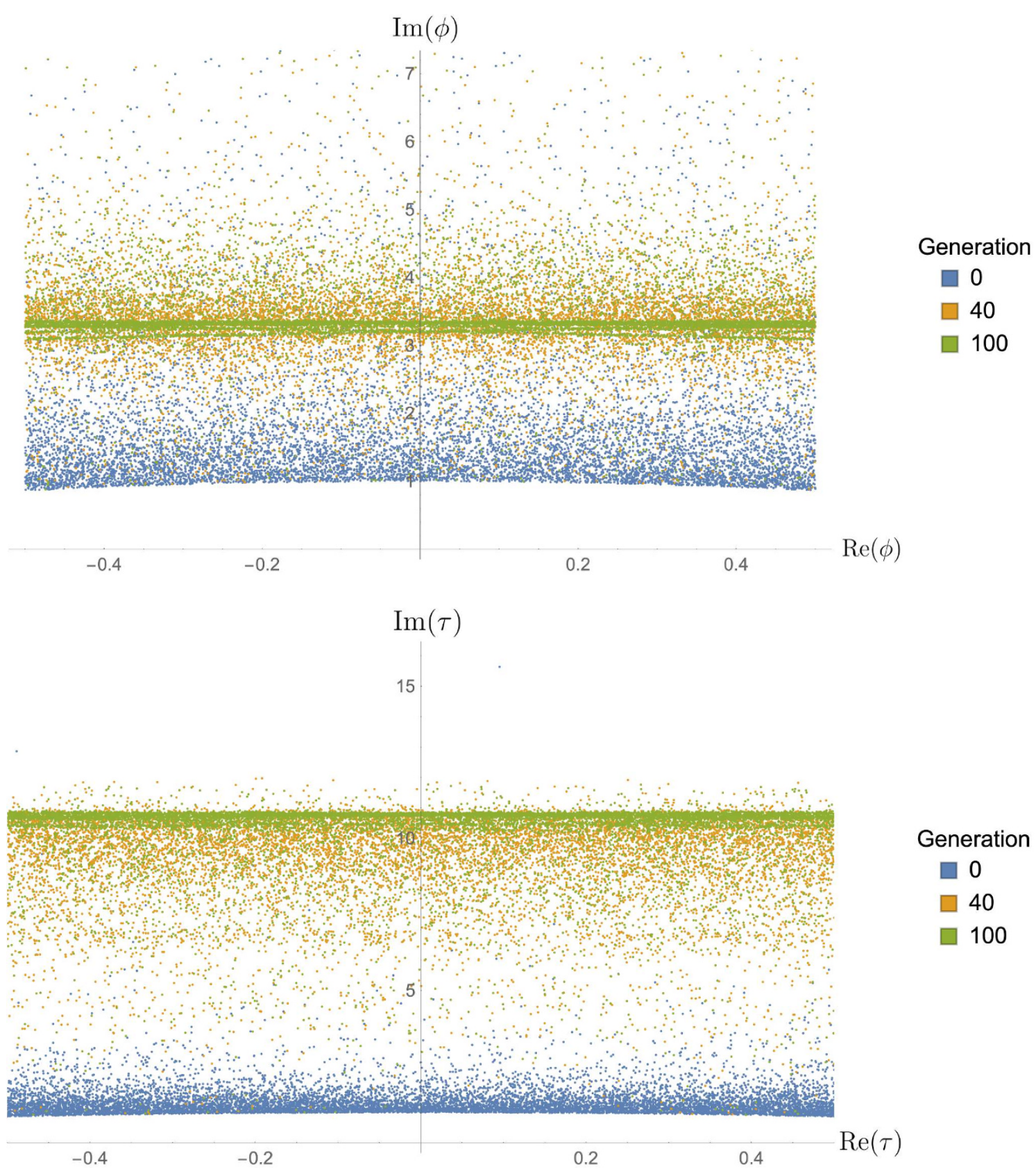

Figure 8. Evolution of the distribution of $\phi$ (top) and $\tau$ (bottom) for three generations.

generations. We employ no crossover within this search since it appears to be more efficient than employing a 2-point crossover operator.

Using the algorithm described in section 3.2, we find an evolution of the initial population as depicted in figures 8 and 9 . Even though we only promoted $g_{\mathrm{s}}$ to be a search parameter, $y=\operatorname{Im}(\tau)$ also accumulates around a value of approximately given by $y \approx 10.68$, cf. the bottom plot of figure 8. As described in the introduction, this is a generic observation for GAs during which the evolution exerts a strong pull on some components of 


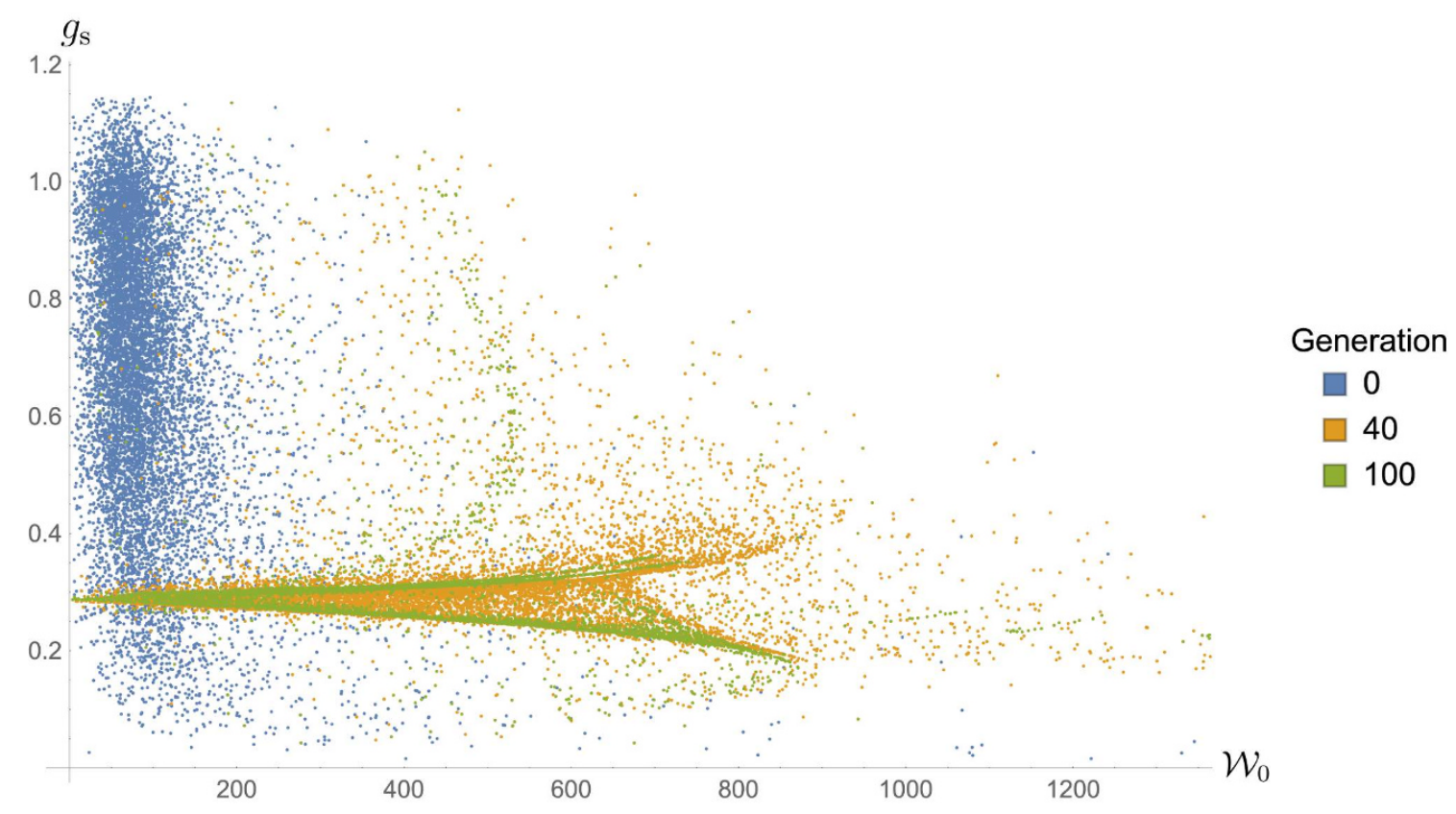

Figure 9. Evolution of the distribution of $g_{\mathrm{s}}$ and $\mathcal{W}_{0}$ for three generations.

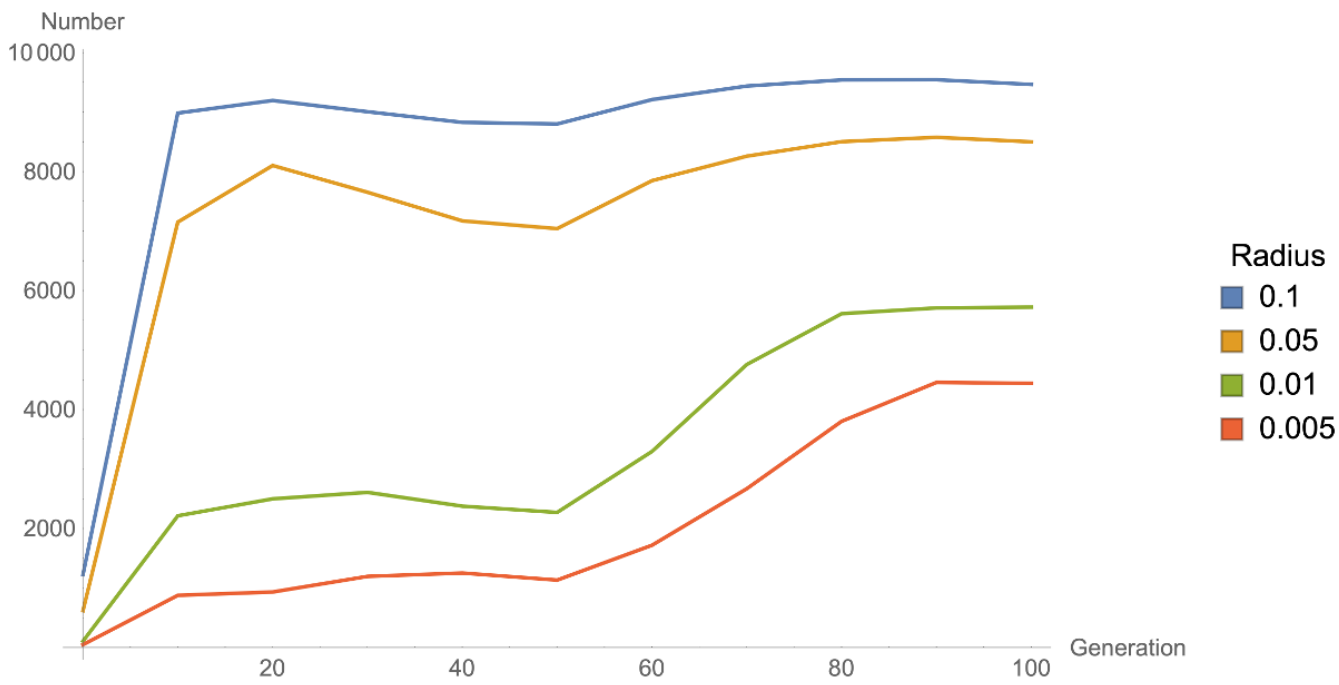

Figure 10. Number of members in neighborhoods of different radius around $g_{\mathrm{s}}^{*}$.

the moduli. In contrast, the values of $\operatorname{Re}(\phi)$ and $\operatorname{Re}(\tau)$ remain equally scattered across the fundamental domain. Remarkably, we detect a correlation between $g_{\mathrm{s}}$ and $\mathcal{W}_{0}$, see figure 9 . For small values of $\mathcal{W}_{0}$, the value of $g_{\mathrm{s}}$ is almost perfectly pinned to $g_{\mathrm{s}}^{*}$, whereas the solutions tend to diverge away from $g_{\mathrm{s}}^{*}$ for large $\mathcal{W}_{0}$. This clearly raises the question whether we can improve on our findings by simultaneously minimizing $\mathcal{W}_{0}$.

To quantify convergence, we count the number of members within neighborhoods of different sizes. The progress of convergence from one generation to another is shown in figure 10. We observe that decreasing $\delta g_{\mathrm{s}}$ after generation 50 clearly pushes the population 

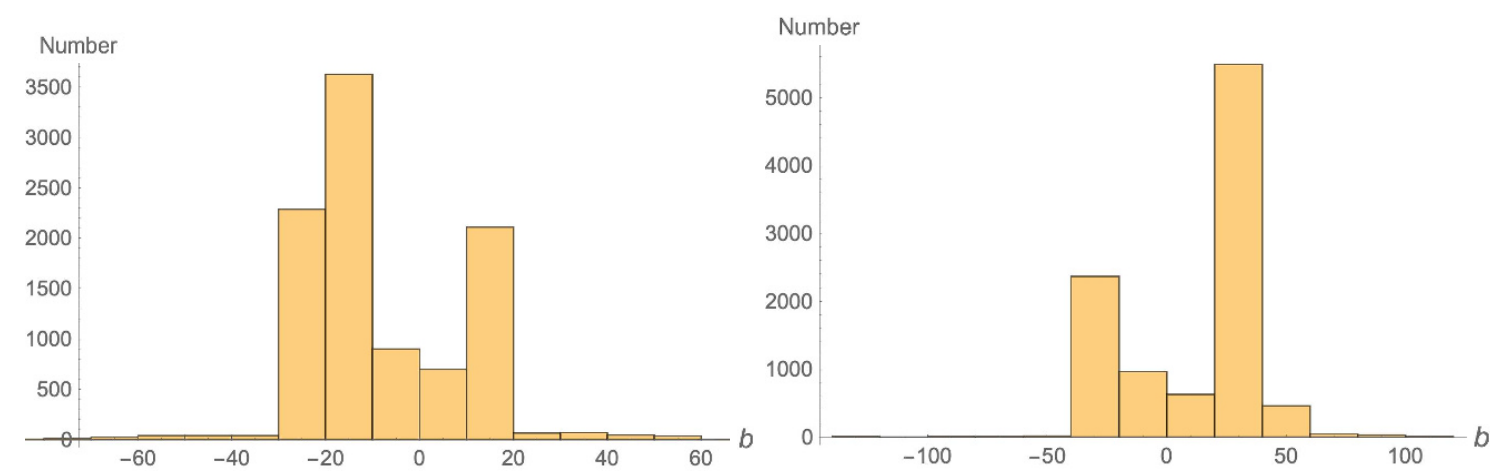

Figure 11. The distribution of $b$ for generation 10 (left) and 30 (right).
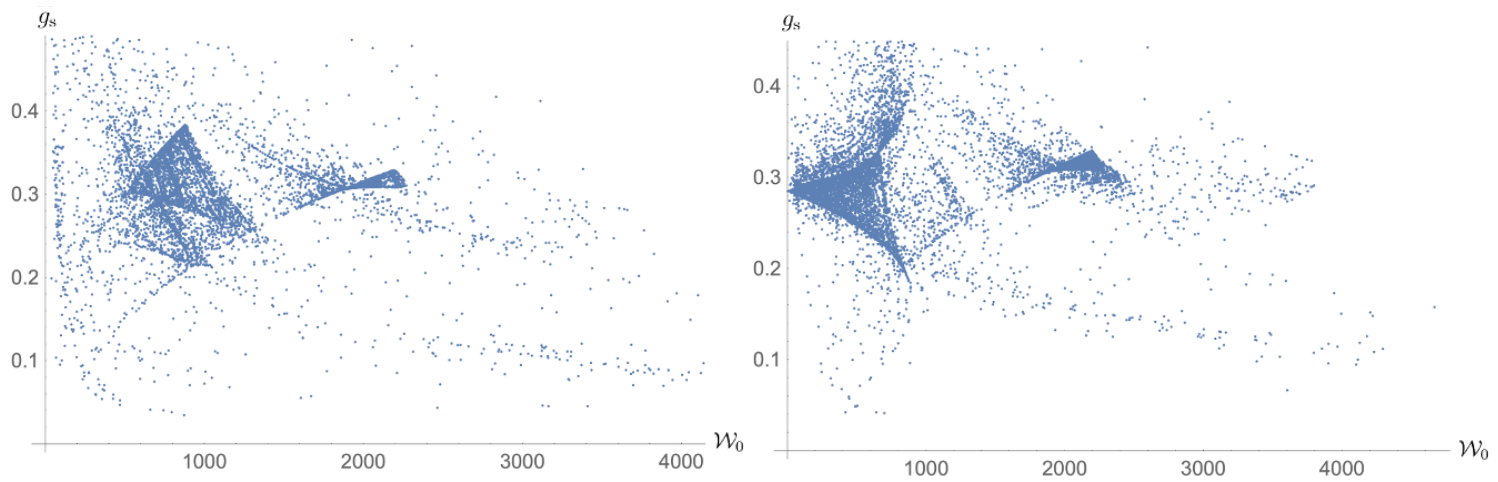

Figure 12. The distribution of vacua in the $\mathcal{W}_{0}-g_{\mathrm{s}}$-plane for generation 10 (left) and 30 (right).

closer to $g_{\mathrm{s}}^{*}$. Moreover, around $90 \%$ of the population can be found within the interval $\left[g_{\mathrm{s}}^{*}-0.1, g_{\mathrm{s}}^{*}+0.1\right]$ after only 10 generations.

As discussed in section 3.3, the initial population evolves rapidly towards a region close to the optimal solution. The drop in blue and orange line observed in figure 10 is associated to the fact that the population gathers around two local fitness maxima. However, the algorithm picks out a global maximum over the course of a few generations. This can also be understood by looking at the distribution of certain fluxes forming the schemata. For simplicity, we only look at one dominating flux. Figure 11 shows that there are two dominating peaks in the distribution of the flux number $b$ at generation 10 (left), recall eq. (5.6). Once the dominating schema wins, the population is pulled towards the peak on the right as depicted on the right hand side of figure 11. This can also be supported by considering the distribution of vacua in the $\mathcal{W}_{0}-g_{\mathrm{s}}$-plane as shown in figure 12 . Notice that briefly the total number of members in the $1 \sigma$-region decreases, cf. figure 10 . It is therefore important to keep in mind that the purpose of the algorithm is not to find as many suitable members as possible, but to maximize the fitness function.

Finally, let us comment on the distribution of fluxes. By performing PCA on the fluxes we observe that there are essentially only two free unconstrained directions in flux space, namely $b_{0}$ and $d_{0}$. The evolution of the corresponding distribution is shown in figure 13 . We observe a triangular shape with two dominating horizontal lines for fixed $d_{0}$. All in all, 


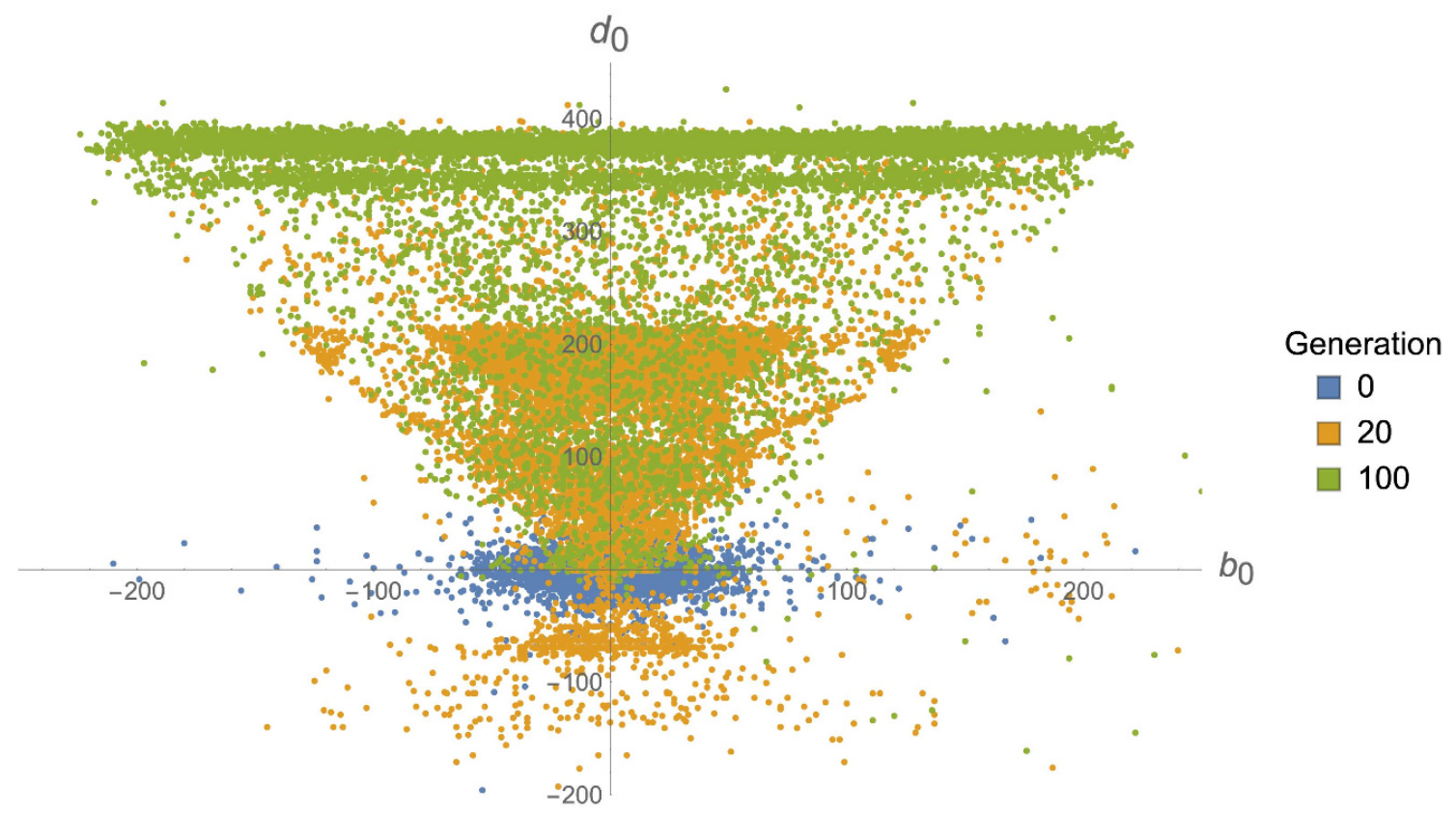

Figure 13. Flux distribution for the two (almost) unconstrained fluxes $b_{0}, d_{0}$ in the final population.

we find that for about $80 \%$ of the population

$$
\begin{aligned}
& a_{0}=1, \quad b_{0} \in[-250,250], \quad c_{0}=0, \quad d_{0} \in[370,390], \\
& a \in[-1,1], \quad b \in[-36,-33], \quad c=-1, \quad d \in[-3,3] .
\end{aligned}
$$

\subsection{Minimizing $\mathcal{W}_{0}$}

Here, we try to find solutions with $\mathcal{W}_{0}=0$. As discussed previously, these solutions have special properties in terms of the fluxes and VEVs. Notice that we do not implement these analytic properties by hand, but simply look for solutions with $P_{1}(\tau)=P_{2}(\tau)=0$ for $\tau$ and physical VEVs. So the algorithm does not know in advance about these special characteristic features associated to $\mathcal{W}_{0}=0$ solutions. We would like to understand whether this has any impact on our genetic algorithm. We consider the parameters

$$
\mathcal{W}_{0}^{*}=0, \quad \delta \mathcal{W}=20, \quad p=10000, \quad N_{\text {gen }}=180, \quad L_{\max }=500, \quad q_{\text {mut }}=0.8
$$

We decrease the support of the fitness function by $50 \%$ at generation 50,70 and 90 .

As shown in figure 14, the populations quickly converge towards small values of $\mathcal{W}_{0}$. However, the rate of convergence is significantly less than in e.g. figure 10. This becomes even more apparent when investigating the FDC of this task. Indeed, the evolution of the FDC in figure 15 shows that, while minimizing itself is not problematic at all, finding $\mathcal{W}_{0}<2.5$ is GA-difficult. For one thing, the lack in convergence is related to the scarcity of $\mathcal{W}_{0}=0$-solutions in comparison to those with $\mathcal{W}_{0} \neq 0$. As derived in [81], the number $N_{\text {vac }}\left(L, \mathcal{W}_{0}=0\right)$ of $\mathcal{W}_{0}=0$-solutions for given flux scale $L \leq L_{\max }$

$$
\frac{N_{\mathrm{vac}}\left(L, \mathcal{W}_{0}=0\right)}{N_{\mathrm{vac}}(L)} \sim \frac{9}{8 \pi^{2}} \frac{\log (L)}{L}
$$




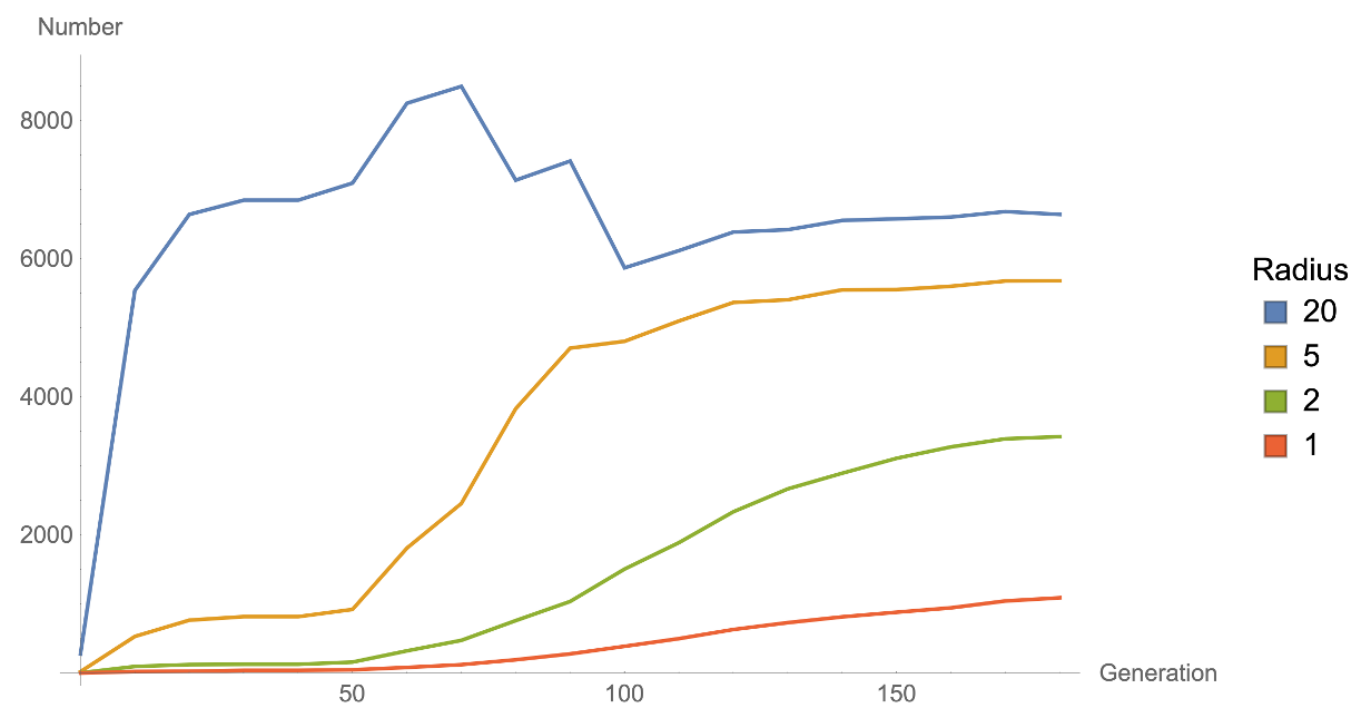

Radius

20

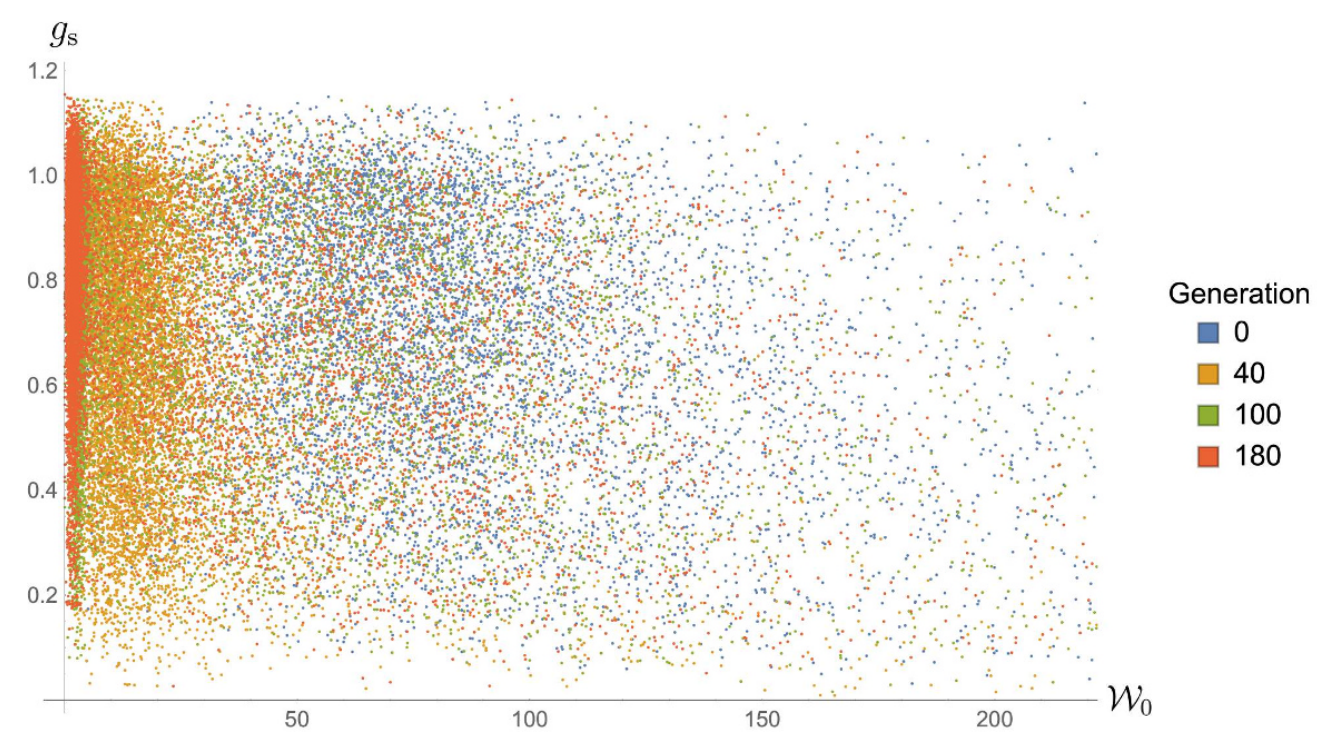

Figure 14. Top: the number of members in neighborhoods of different radius around $\mathcal{W}_{0}^{*}$. Bottom: the evolution of the distribution of $g_{\mathrm{s}}$ and $\mathcal{W}_{0}$ for four generations.

in terms of the total number of vacua $N_{\text {vac }}(L)$. On top of that, at small $L$ discretization effects overshadow $\mathcal{W}_{0}=0$ solutions (see for example figure 3 in [81]) which is why we have to go to comparatively large $L$. In any case, we observe that special solutions with $\mathcal{W}_{0}=0$ are difficult for our algorithm to identify. Notwithstanding, we note that a small fraction of individuals do satisfy $\mathcal{W}_{0}=0$.

In the light of the ongoing discussion about KKLT and related dS-vacua constructions $[114,115]$, it is crucial to search the landscape for suitable flux vacua, see e.g. [99, 116] for previous attempts. In order to safely ignore perturbative corrections, it is necessary to have $\mathcal{W}_{0} \ll 1[101,117]$. One can infer the existence of such vacua by employing statistical arguments [8]. However, it is generally true that these vacua are less common than those with $\mathcal{W}_{0} \gtrsim \mathcal{O}(1)$ using similar arguments to the one above [10]. 


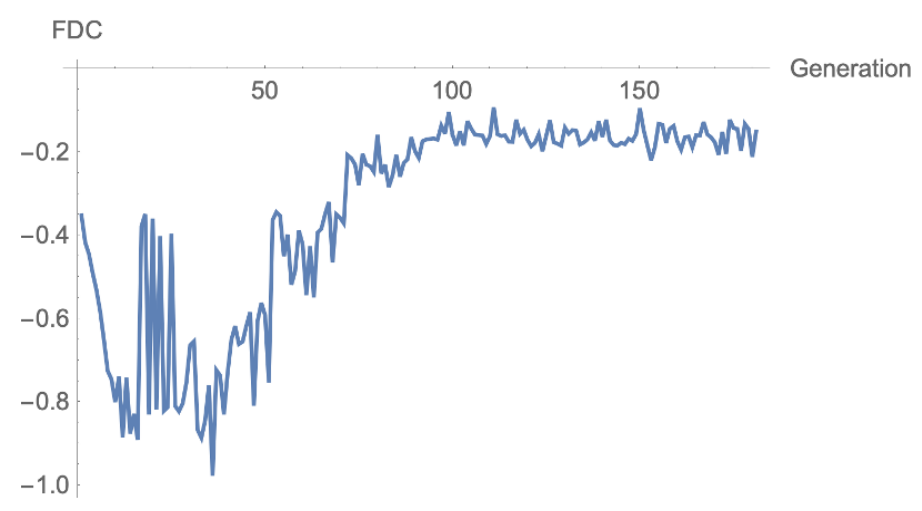

Figure 15. Fitness distance correlation as a function of generation.

In our case, the smallest value found via the above algorithm is $\mathcal{W}_{0} \sim 10^{-2}$. Here it is important to keep in mind that the symmetric $T^{6}$ is only a simple toy example. General Calabi-Yau orientifolds with many moduli are expected to have a more involved structure within their flux landscape. Therefore, it would be interesting to see whether smaller values of $\mathcal{W}_{0}$ are obtainable within such geometries utilizing GAs and extensions thereof.

\section{$5.4 \quad$ Fixing mass scales}

To conclude our survey of applications, we investigate a more difficult problem in the context of GAs. Namely, we elaborate on the idea of specifying several observables within our search. As we will see further below, it is necessary to try different selection methods in order to guarantee the success of our GA. We restrict our analysis to the case of no crossover which allows us to study the impact of different selection methods more easily.

The question of finding flux configurations with certain values of the mass scales is ubiquitous in the context of model building in string theory. For simplicity, we compute the diagonal entries of the mass matrix (2.8) for the canonically normalized fields evaluated at the SUSY minimum $D_{\phi} \mathcal{W}=D_{\tau} \mathcal{W}=0$ which we denote $M_{\operatorname{Re}(\phi)}$ etc. We fix all four masses at the same value, i.e., we consider

$$
M^{*}=5000, \quad \delta M=1000, \quad p=10000, \quad N_{\text {gen }}=120, \quad q_{\text {mut }}=1 .
$$

We determined the associated weights and the offset in (3.2) by trial and error, giving

$$
w_{\operatorname{Re}(\phi)}=6.8, \quad w_{\operatorname{Im}(\phi)}=1.0, \quad w_{\operatorname{Re}(\tau)}=1.3, \quad w_{\operatorname{Im}(\tau)}=0.4, \quad b=0 .
$$

We decrease $\delta M$ only once at generation 60 .

Figure 16 summarizes the results obtained by running the GA. The left plot shows that certain quantities are treated differently from the others during the algorithm's evolution. The right hand side of figure 16 depicts the number of individuals that satisfy the search criteria for all four mass scales at the same time. In comparison to the results obtained in the previous section, we conclude that it is significantly harder to get closer to the optimal solution whenever several parameters are taken into account. However, this might simply be related to a bad choice of selection methods. As mentioned previously, many of such 

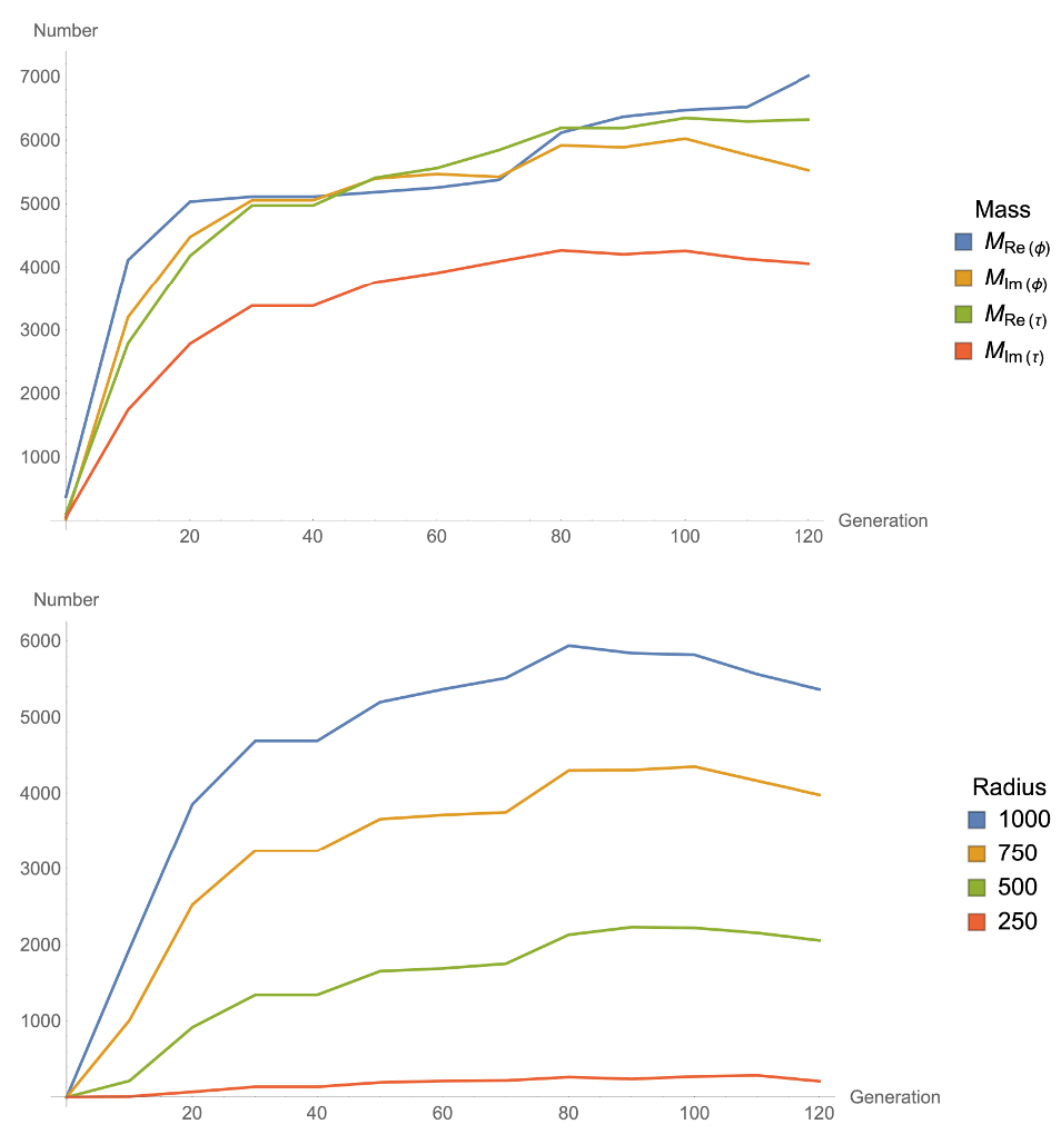

Figure 16. Top: the number of individuals having a single mass matrix entry within a neighborhood of $r=500$. Bottom: the number of individuals having all four masses within neighborhoods of different radii.

methods have been discussed in the literature, but we restricted mainly to the so-called roulette-wheel selection so far where the fitness itself is used as a measure of probability to procreate. This typically leads to premature convergence towards a local fitness maximum. This is not really problematic in the case of one GA-parameter. In the context of several, however, this is a serious issue.

Therefore, we tested different selection techniques such as

- rank-weighted selection where each member is ranked by a number $1, \ldots, p$ according to its fitness. This ranking is used to determine the probability for procreation which is chosen to be a linear mapping here. In comparison to roulette-wheel selection this means that individuals with high fitness do not dominate the crossover procedure too early during evolution.

- tournament selection where so-called tournaments between a subset of members are performed. That is, we pick $k<p$ individuals from our population and take only the fittest for breeding. Since we need to make several of such tournaments, the diversity of fit individuals in the descendant population is enhanced. In other words, the population does not localize too early around a local fitness maximum. 

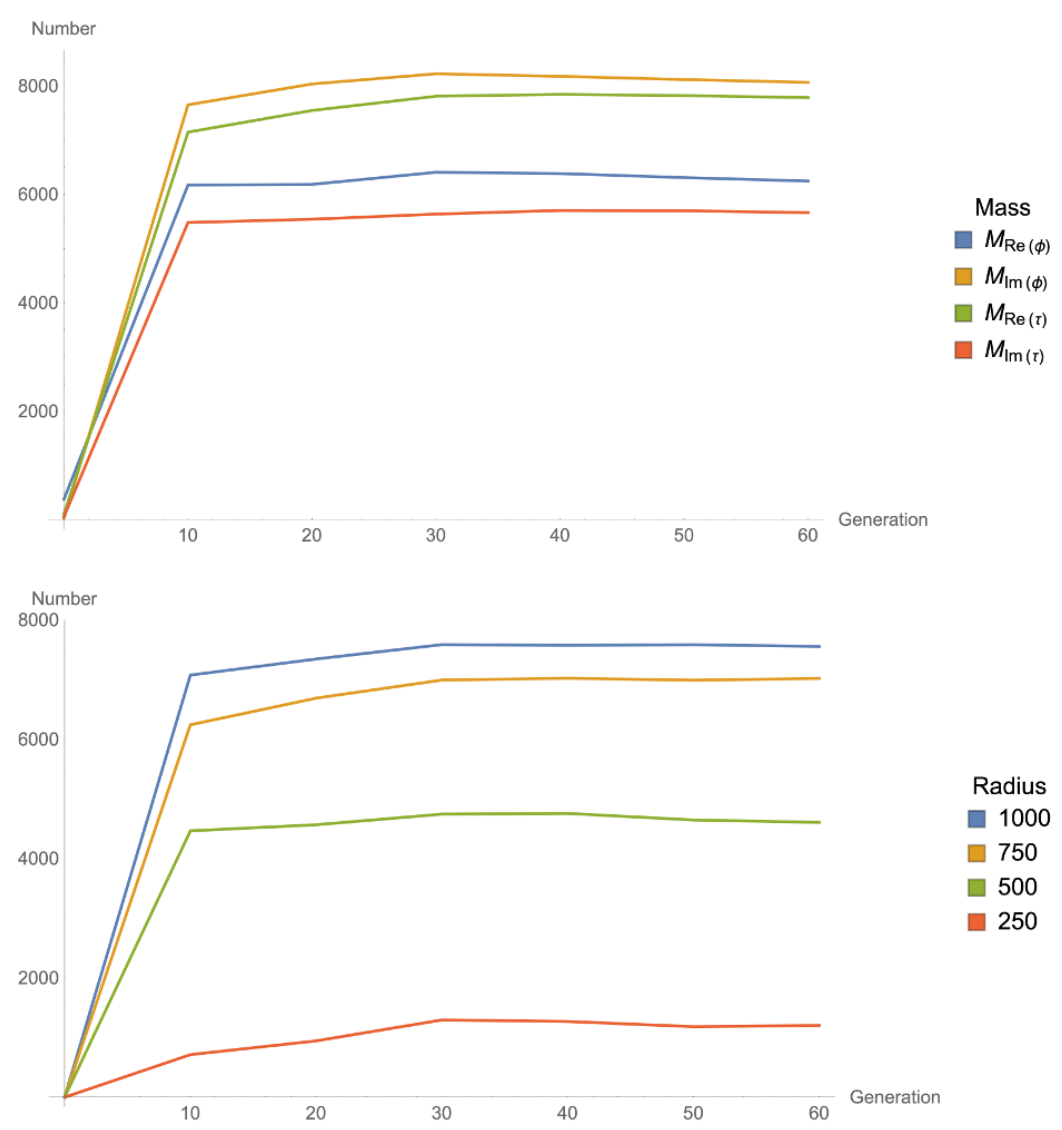

Figure 17. Top: the number of individuals having a single mass matrix entry within a neighborhood of $r=500$ using tournament selection. Bottom: the number of individuals having all four masses within different neighborhoods.

The first does not lead to any better results, but is significantly slower in terms of convergence around the optimal solution. In contrast, tournament selection is really efficient in determining a global fitness maximum that satisfies the constraints on all search parameters.

Starting with the same initial population as above, we use the parameters

$$
w_{\operatorname{Re}(\phi)}=w_{\operatorname{Im}(\phi)}=w_{\operatorname{Re}(\tau)}=w_{\operatorname{Im}(\tau)}=1.0, \quad b=0
$$

and consider only $N_{\text {gen }}=60$ generations. The results for tournament selection are summarized in figure 17. A crucial advantage of using tournament selection is that one can achieve significantly better results compared to figure 16 with only half the number of generations. Even though performing several tournaments is computationally more expensive, this selection method is therefore more efficient in the scenario under consideration.

Finally, we would like to discuss tasks that are hard to tackle with GAs. In the context of this section, we observed some difficulty trying to arrange mass hierarchies. We believe that this is related to the more general circumstance that, whenever observables are strongly correlated, it is hard to fix them simultaneously. In the worst case, certain parameter values might be incompatible with each other, in the sense that no such pair exists in the fitness 
landscape. Alternatively, such solutions are very scarce. Identifying these limitations is typically beyond the GA's (or any stochastic search algorithm's) capabilities.

\section{Conclusions}

In this paper, we demonstrated that genetic algorithms are a helpful tool for searching for specific phenomenological features in the landscape of flux vacua. This is possible due to the underlying structure of the landscape, which is crucial for the success of GAs. We showed that GAs may be used not only to speed up the search for phenomenologically interesting models, but also to uncover unknown structures and correlations in the landscape.

In the present work, we discussed various properties of GAs that allow for a systematic and efficient study of the flux landscape. Specifically, we proposed a dictionary between concepts in these two respective fields that could be useful for future applications of GAs to string theory.

Applying our dictionary, we first considered the example of a Calabi-Yau hypersurface in a weighted projective space close to a conifold point. We observed the important effect the mutation rate has on the algorithm's performance, and the need to adjust it for various applications. We also studied the general dynamics of the GA, with an initial period of strong pull towards a local optimum followed by slower convergence to the global optimum. Along these lines, we used TDA and PCA to study the "shape" of the distribution of fluxes as a function of generation. These methods confirmed our ideas regarding clustering in the final population. We showed that distinct crossover operations typically lead to different evolutionary properties of the GA, albeit the final population turns out to be almost equivalent. In addition, we compared various breeding mechanisms which could be useful in scaled-up versions of the models investigated in this paper. In contrast to a Metropolis algorithm, the GA identifies links between various vacua due to the applied crossover procedure, enabling it to exploit the landscape's structure. On the other hand, a Metropolis algorithm features no such benefits.

We also applied the GA to a symmetric $T^{6}$. While searches for values of the string coupling are GA-easy, we showed that finding solutions satisfying $\mathcal{W}_{0}=0$ is difficult in the context of GAs. Although these solutions emerged within our searches, their scarcity seems to counteract the formation of associated schemata. This behavior can be quantified in terms of the fitness distance correlation. Finally and most importantly, we demonstrated that GAs are capable of finding vacua with several properties, namely specifying several masses. This success is encouraging as we look towards phenomenologically-viable constructions.

We emphasize that the applications of GAs are diverse and can easily be translated into other promising setups of, e.g., model building in string theory. In the future, we would like to turn to phenomenologically interesting questions such as testing the WGC or other statements about the landscape. We would also like to investigate different selection methods and other GA-relevant techniques allowing for an improved version of the algorithm applied in this paper. 


\section{Acknowledgments}

We thank Arthur Hebecker and Pablo Soler for discussion. This work is supported in part by the DOE grant DE-SC0017647 and the Kellett Award of the University of Wisconsin. AC would like to thank the Straka Fund at UW-Madison for financial support. GS would like to thank the Aspen Center for Physics for hospitality during the final stages of this work. AS acknowledges support by the German Academic Scholarship Foundation and the hospitality of the University of Wisconsin during part of this work.

Open Access. This article is distributed under the terms of the Creative Commons Attribution License (CC-BY 4.0), which permits any use, distribution and reproduction in any medium, provided the original author(s) and source are credited.

\section{References}

[1] R. Bousso and J. Polchinski, Quantization of four form fluxes and dynamical neutralization of the cosmological constant, JHEP 06 (2000) 006 [hep-th/0004134] [INSPIRE].

[2] L. Susskind, The Anthropic landscape of string theory, hep-th/0302219 [INSPIRE].

[3] M.R. Douglas, The Statistics of string/M theory vacua, JHEP 05 (2003) 046 [hep-th/0303194] [INSPIRE].

[4] T. Banks, M. Dine and E. Gorbatov, Is there a string theory landscape?, JHEP 08 (2004) 058 [hep-th/0309170] [INSPIRE].

[5] T. Banks, Landskepticism or why effective potentials don't count string models, 2004, hep-th/0412129 [INSPIRE].

[6] T. Banks, The Top $10^{500}$ Reasons Not to Believe in the Landscape, arXiv:1208.5715 [INSPIRE].

[7] S. Ashok and M.R. Douglas, Counting flux vacua, JHEP 01 (2004) 060 [hep-th/0307049] [INSPIRE].

[8] F. Denef and M.R. Douglas, Distributions of flux vacua, JHEP 05 (2004) 072 [hep-th/0404116] [INSPIRE].

[9] M.R. Douglas, B. Shiffman and S. Zelditch, Critical points and supersymmetric vacua, Commun. Math. Phys. 252 (2004) 325 [math/0402326] [INSPIRE].

[10] F. Denef and M.R. Douglas, Distributions of nonsupersymmetric flux vacua, JHEP 03 (2005) 061 [hep-th/0411183] [INSPIRE].

[11] L. Susskind, Supersymmetry breaking in the anthropic landscape, hep-th/0405189 [INSPIRE].

[12] M.R. Douglas, Statistical analysis of the supersymmetry breaking scale, hep-th/0405279 [INSPIRE].

[13] M. Dine, E. Gorbatov and S.D. Thomas, Low energy supersymmetry from the landscape, JHEP 08 (2008) 098 [hep-th/0407043] [INSPIRE].

[14] J.P. Conlon and F. Quevedo, On the explicit construction and statistics of Calabi-Yau flux vacua, JHEP 10 (2004) 039 [hep-th/0409215] [INSPIRE]. 
[15] R. Kallosh and A.D. Linde, Landscape, the scale of SUSY breaking and inflation, JHEP 12 (2004) 004 [hep-th/0411011] [INSPIRE].

[16] F. Marchesano, G. Shiu and L.-T. Wang, Model building and phenomenology of flux-induced supersymmetry breaking on D3-branes, Nucl. Phys. B 712 (2005) 20 [hep-th/0411080] [INSPIRE].

[17] M. Dine, D. O'Neil and Z. Sun, Branches of the landscape, JHEP 07 (2005) 014 [hep-th/0501214] [INSPIRE].

[18] B.S. Acharya, F. Denef and R. Valandro, Statistics of M-theory vacua, JHEP 06 (2005) 056 [hep-th/0502060] [INSPIRE].

[19] K.R. Dienes, Statistics on the heterotic landscape: Gauge groups and cosmological constants of four-dimensional heterotic strings, Phys. Rev. D 73 (2006) 106010 [hep-th/0602286] [INSPIRE].

[20] F. Gmeiner, R. Blumenhagen, G. Honecker, D. Lüst and T. Weigand, One in a billion: MSSM-like D-brane statistics, JHEP 01 (2006) 004 [hep-th/0510170] [INSPIRE].

[21] M.R. Douglas and W. Taylor, The Landscape of intersecting brane models, JHEP 01 (2007) 031 [hep-th/0606109] [INSPIRE].

[22] C. Vafa, The String landscape and the swampland, hep-th/0509212 [INSPIRE].

[23] H. Ooguri and C. Vafa, On the Geometry of the String Landscape and the Swampland, Nucl. Phys. B 766 (2007) 21 [hep-th/0605264] [INSPIRE].

[24] E. Palti, The Swampland: Introduction and Review, Fortsch. Phys. 67 (2019) 1900037 [arXiv: 1903.06239] [INSPIRE].

[25] A. Cole and G. Shiu, Topological Data Analysis for the String Landscape, JHEP 03 (2019) 054 [arXiv: 1812.06960] [INSPIRE].

[26] Y.-H. He, Deep-Learning the Landscape, arXiv:1706.02714 [InSPIRE].

[27] D. Krefl and R.-K. Seong, Machine Learning of Calabi-Yau Volumes, Phys. Rev. D 96 (2017) 066014 [arXiv: 1706.03346] [INSPIRE].

[28] F. Ruehle, Evolving neural networks with genetic algorithms to study the String Landscape, JHEP 08 (2017) 038 [arXiv:1706.07024] [INSPIRE].

[29] J. Carifio, J. Halverson, D. Krioukov and B.D. Nelson, Machine Learning in the String Landscape, JHEP 09 (2017) 157 [arXiv: 1707.00655] [INSPIRE].

[30] Y.-N. Wang and Z. Zhang, Learning non-Higgsable gauge groups in $4 D$ F-theory, JHEP 08 (2018) 009 [arXiv: 1804.07296] [INSPIRE].

[31] K. Bull, Y.-H. He, V. Jejjala and C. Mishra, Machine Learning CICY Threefolds, Phys. Lett. B 785 (2018) 65 [arXiv:1806.03121] [INSPIRE].

[32] D. Klaewer and L. Schlechter, Machine Learning Line Bundle Cohomologies of Hypersurfaces in Toric Varieties, Phys. Lett. B 789 (2019) 438 [arXiv:1809.02547] [INSPIRE].

[33] A. Mütter, E. Parr and P.K.S. Vaudrevange, Deep learning in the heterotic orbifold landscape, Nucl. Phys. B 940 (2019) 113 [arXiv:1811.05993] [InSPIRE].

[34] J. Halverson, B. Nelson and F. Ruehle, Branes with Brains: Exploring String Vacua with Deep Reinforcement Learning, JHEP 06 (2019) 003 [arXiv: 1903.11616] [INSPIRE]. 
[35] Y.-H. He and S.-J. Lee, Distinguishing Elliptic Fibrations with AI, arXiv:1904.08530 [INSPIRE].

[36] J. Holland, Adaptation in Natural and Artificial Systems, The MIT Press reprinted (1992).

[37] E. David, Genetic Algorithms in Search, Optimization and Machine Learning, Addison-Wesley, (1989).

[38] J. Holland, The Royal Road for Genetic Algorithms: Fitness Landscapes and GA Performance, MIT Press (1992).

[39] C. Reeves and J. Rowe, Genetic Algorithms: Principles and Perspectives, Springer (2002).

[40] R. Haupt, Practical genetic algorithms, Wyley (2004).

[41] D. Michalewicz, How to Solve It: Modern Heuristics, Springer (2004).

[42] B.C. Allanach, D. Grellscheid and F. Quevedo, Genetic algorithms and experimental discrimination of SUSY models, JHEP 07 (2004) 069 [hep-ph/0406277] [INSPIRE].

[43] Y. Akrami, P. Scott, J. Edsjo, J. Conrad and L. Bergstrom, A Profile Likelihood Analysis of the Constrained MSSM with Genetic Algorithms, JHEP 04 (2010) 057 [arXiv:0910.3950] [INSPIRE].

[44] S. Abel, D.G. Cerdeño and S. Robles, The Power of Genetic Algorithms: what remains of the pMSSM?, arXiv:1805.03615 [inSPIRE].

[45] T.S. Metcalfe, R.E. Nather and D.E. Winget, Genetic-algorithm-based asteroseismological analysis of the dbv white dwarf gd 358, Astrophys. J. 545 (2000) 974 [astro-ph/0008022] [INSPIRE].

[46] M.R. Mokiem, A. de Koter, J. Puls, A. Herrero, F. Najarro and M.R. Villamariz, Spectral analysis of early-type stars using a genetic algorithm based fitting method, Astron. Astrophys. 441 (2005) 711 [astro-ph/0506751] [INSPIRE].

[47] V. Rajpaul, Genetic algorithms in astronomy and astrophysics, in Proceedings, 56th Annuall Conference of the South African Institute of Physics (SAIP 2011), Gauteng, South Africa, 12-15 July 2011, pp. 519-524, (2012) [arXiv:1202.1643] [INSPIRE].

[48] S. Nesseris and J. García-Bellido, A new perspective on Dark Energy modeling via Genetic Algorithms, JCAP 11 (2012) 033 [arXiv:1205.0364] [INSPIRE].

[49] R. Hogan, M. Fairbairn and N. Seeburn, GAz: A Genetic Algorithm for Photometric Redshift Estimation, Mon. Not. Roy. Astron. Soc. 449 (2015) 2040 [arXiv:1412.5997] [INSPIRE].

[50] J. Blåbäck, U. Danielsson and G. Dibitetto, Fully stable $d S$ vacua from generalised fluxes, JHEP 08 (2013) 054 [arXiv: 1301.7073] [INSPIRE].

[51] C. Damian, L.R. Diaz-Barron, O. Loaiza-Brito and M. Sabido, Slow-Roll Inflation in Non-geometric Flux Compactification, JHEP 06 (2013) 109 [arXiv:1302.0529] [INSPIRE].

[52] C. Damian and O. Loaiza-Brito, More stable de Sitter vacua from S-dual nongeometric fluxes, Phys. Rev. D 88 (2013) 046008 [arXiv:1304.0792] [INSPIRE].

[53] J. Blåbäck, U. Danielsson and G. Dibitetto, Accelerated Universes from type IIA Compactifications, JCAP 03 (2014) 003 [arXiv:1310.8300] [INSPIRE].

[54] J. Blåbäck, D. Roest and I. Zavala, de Sitter Vacua from Nonperturbative Flux Compactifications, Phys. Rev. D 90 (2014) 024065 [arXiv:1312.5328] [INSPIRE]. 
[55] S. Abel and J. Rizos, Genetic Algorithms and the Search for Viable String Vacua, JHEP 08 (2014) 010 [arXiv: 1404.7359] [INSPIRE].

[56] F. Denef and M.R. Douglas, Computational complexity of the landscape. I., Annals Phys. 322 (2007) 1096 [hep-th/0602072] [INSPIRE].

[57] N. Bao, R. Bousso, S. Jordan and B. Lackey, Fast optimization algorithms and the cosmological constant, Phys. Rev. D 96 (2017) 103512 [arXiv:1706.08503] [InSPIRE].

[58] F. Denef, M.R. Douglas, B. Greene and C. Zukowski, Computational complexity of the landscape II - Cosmological considerations, Annals Phys. 392 (2018) 93 [arXiv: 1706. 06430] [INSPIRE].

[59] J. Halverson and F. Ruehle, Computational Complexity of Vacua and Near-Vacua in Field and String Theory, Phys. Rev. D 99 (2019) 046015 [arXiv: 1809.08279] [INSPIRE].

[60] D. Silver et al., Mastering the game of go with deep neural networks and tree search, Nature 529 (2016) 484.

[61] T. Salimans, J. Ho, X. Chen, S. Sidor and I. Sutskever, Evolution Strategies as a Scalable Alternative to Reinforcement Learning, arXiv:1703.03864.

[62] N. Arkani-Hamed, L. Motl, A. Nicolis and C. Vafa, The String landscape, black holes and gravity as the weakest force, JHEP 06 (2007) 060 [hep-th/0601001] [INSPIRE].

[63] J. Brown, W. Cottrell, G. Shiu and P. Soler, Fencing in the Swampland: Quantum Gravity Constraints on Large Field Inflation, JHEP 10 (2015) 023 [arXiv: 1503.04783] [INSPIRE].

[64] J. Brown, W. Cottrell, G. Shiu and P. Soler, On Axionic Field Ranges, Loopholes and the Weak Gravity Conjecture, JHEP 04 (2016) 017 [arXiv: 1504.00659] [INSPIRE].

[65] B. Heidenreich, M. Reece and T. Rudelius, Sharpening the Weak Gravity Conjecture with Dimensional Reduction, JHEP 02 (2016) 140 [arXiv: 1509.06374] [INSPIRE].

[66] B. Heidenreich, M. Reece and T. Rudelius, Evidence for a sublattice weak gravity conjecture, JHEP 08 (2017) 025 [arXiv: 1606.08437] [INSPIRE].

[67] M. Montero, G. Shiu and P. Soler, The Weak Gravity Conjecture in three dimensions, JHEP 10 (2016) 159 [arXiv:1606.08438] [INSPIRE].

[68] S. Andriolo, D. Junghans, T. Noumi and G. Shiu, A Tower Weak Gravity Conjecture from Infrared Consistency, Fortsch. Phys. 66 (2018) 1800020 [arXiv:1802.04287] [INSPIRE].

[69] P. Charbonneau, Genetic Algorithms in Astronomy and Astrophysics, Astrophys. J. Suppl. $101(1995) 309$.

[70] P. Charbonneau and B. Knapp, A user's guide to pikaia 1.0, Tech. Rep. TN-418+IA, National Center for Atmospheric Research (1995).

[71] P. Charbonneau, An introduction to genetic algorithms for numerical optimization, NCAR Technical Note (2002) 74 .

[72] P. Charbonneau, Release notes for pikaia 1.2, Tech. Rep. TN-451+STR, National Center for Atmospheric Research (2002).

[73] T. Jones and S. Forrest, Fitness distance correlation as a measure of problem difficulty for genetic algorithms, in Proc. 6th Int. Conf. on Genetic Algorithms, pp. 184-192 (1995).

[74] P. Collard, A. Gaspar, M. Clergue and C. Escazut, Fitness distance correlation, as statistical measure of genetic algorithm difficulty, revisited, in ECAI, pp. 650-654, Citeseer (1998). 
[75] J.D. Bryngelson, J.N. Onuchic, N.D. Socci and P.G. Wolynes, Funnels, pathways, and the energy landscape of protein folding: a synthesis, Proteins 21 (1995) 167.

[76] J. Khoury and O. Parrikar, Search Optimization, Funnel Topography and Dynamical Criticality on the String Landscape, arXiv:1907.07693 [INSPIRE].

[77] W. Ruml, J.T. Ngo, J. Marks and S.M. Shieber, Easily searched encodings for number partitioning, J. Optim. Theory. Appl. 89 (1996) 251.

[78] M. Graña, Flux compactifications in string theory: A Comprehensive review, Phys. Rept. 423 (2006) 91 [hep-th/0509003] [INSPIRE].

[79] M.R. Douglas and S. Kachru, Flux compactification, Rev. Mod. Phys. 79 (2007) 733 [hep-th/0610102] [INSPIRE].

[80] S.B. Giddings, S. Kachru and J. Polchinski, Hierarchies from fluxes in string compactifications, Phys. Rev. D 66 (2002) 106006 [hep-th/0105097] [INSPIRE].

[81] O. DeWolfe, A. Giryavets, S. Kachru and W. Taylor, Enumerating flux vacua with enhanced symmetries, JHEP 02 (2005) 037 [hep-th/0411061] [INSPIRE].

[82] S. Gukov, C. Vafa and E. Witten, CFT's from Calabi-Yau four folds, Nucl. Phys. B 584 (2000) 69 [Erratum ibid. B 608 (2001) 477] [hep-th/9906070] [INSPIRE].

[83] J.E. Baker, Reducing bias and inefficiency in the selection algorithm, in Proceedings of the second international conference on genetic algorithms, vol. 206, pp. 14-21 (1987).

[84] D.E. Goldberg, Genetic algorithms in search, Optimization, and MachineLearning, Addison-Wesley Longman Publishing Co., Inc. (1989).

[85] L.D. Whitley et al., The genitor algorithm and selection pressure: why rank-based allocation of reproductive trials is best, in Icga, vol. 89, pp. 116-123, Fairfax, VA (1989).

[86] E. Saliby, Descriptive sampling: a better approach to monte carlo simulation, J. Oper. Res. Soc. 41 (1990) 1133.

[87] D.E. Goldberg and K. Deb, A comparative analysis of selection schemes used in genetic algorithms, in Foundations of genetic algorithms, vol. 1, pp. 69-93, Elsevier (1991).

[88] H. Mühlenbein and D. Schlierkamp-Voosen, The science of breeding and its application to the breeder genetic algorithm, Evol. Comput. 1 (1994) 335.

[89] P.J. Hancock, An empirical comparison of selection methods in evolutionary algorithms, in AISB Workshop on Evolutionary Computing, pp. 80-94, Springer (1994).

[90] P.J. Hancock, Selection methods for evolutionary algorithms, Practical Handbook of Genetic Algorithms 2 (1995) 67.

[91] T. Blickle and L. Thiele, A comparison of selection schemes used in evolutionary algorithms, Evol. Comput. 4 (1996) 361.

[92] D. Thierens, Selection schemes, elitist recombination, and selection intensity, vol. 1998, Utrecht University: Information and Computing Sciences (1998).

[93] S.L. Lohr, Sampling: Design and analysis, CRC Press(1999).

[94] G. Rudolph, Takeover times and probabilities of non-generational selection rules, in Proceedings of the 2nd Annual Conference on Genetic and Evolutionary Computation, pp. 903-910, Morgan Kaufmann Publishers Inc. (2000). 
[95] G. Rudolph, Takeover times of noisy non-generational selection rules that undo extinction, in Artificial Neural Nets and Genetic Algorithms, pp. 268-271, Springer (2001).

[96] C. Reeves and J. Rowe, Genetic Algorithms: Principles and Perspectives: A Guide to GA Theory, vol. 20, Springer Science \& Business Media (2002).

[97] R.L. Haupt and S. Ellen Haupt, Practical genetic algorithms, John Wiley \& Sons, Inc. (2004).

[98] P. Betzler and E. Plauschinn, Type IIB flux vacua and tadpole cancellation, Fortsch. Phys. 67 (2019) 1900065 [arXiv: 1905.08823] [INSPIRE].

[99] A. Giryavets, S. Kachru, P.K. Tripathy and S.P. Trivedi, Flux compactifications on Calabi-Yau threefolds, JHEP 04 (2004) 003 [hep-th/0312104] [INSPIRE].

[100] A. Giryavets, S. Kachru and P.K. Tripathy, On the taxonomy of flux vacua, JHEP 08 (2004) 002 [hep-th/0404243] [INSPIRE].

[101] S. Kachru, R. Kallosh, A.D. Linde and S.P. Trivedi, de Sitter vacua in string theory, Phys. Rev. D 68 (2003) 046005 [hep-th/0301240] [INSPIRE].

[102] V. Balasubramanian, P. Berglund, J.P. Conlon and F. Quevedo, Systematics of moduli stabilisation in Calabi-Yau flux compactifications, JHEP 03 (2005) 007 [hep-th/0502058] [INSPIRE].

[103] A. Westphal, de Sitter string vacua from Kähler uplifting, JHEP 03 (2007) 102 [hep-th/0611332] [INSPIRE].

[104] M. Cirafici, Persistent Homology and String Vacua, JHEP 03 (2016) 045 [arXiv: 1512.01170] [INSPIRE].

[105] H. Edelsbrunner, D. Letscher and A. Zomorodian, Topological persistence and simplification, in Proceedings 41st Annual Symposium on Foundations of Computer Science, pp. 454-463, IEEE (2000).

[106] A. Zomorodian and G. Carlsson, Computing persistent homology, Discrete Comput. Geom. 33 (2005) 249.

[107] G. Carlsson, Topology and data, Bull. Am. Math. Soc. 46 (2009) 255.

[108] G. Carlsson, Topological pattern recognition for point cloud data, Acta Numer. 23 (2014) 289.

[109] A. Cole and G. Shiu, Persistent Homology and Non-Gaussianity, JCAP 03 (2018) 025 [arXiv: 1712.08159] [INSPIRE].

[110] J. Murugan and D. Robertson, An Introduction to Topological Data Analysis for Physicists: From LGM to FRBs, arXiv: 1904.11044 [INSPIRE].

[111] N. Metropolis, A.W. Rosenbluth, M.N. Rosenbluth, A.H. Teller and E. Teller, Equation of state calculations by fast computing machines, J. Chem. Phys. 21 (1953) 1087 [INSPIRE].

[112] S. Kirkpatrick, C. Gelatt and M. Vecchi, Simulated annealing methods, J. Stat. Phys 34 (1984) 975 .

[113] S. Kachru, M.B. Schulz and S. Trivedi, Moduli stabilization from fluxes in a simple IIB orientifold, JHEP 10 (2003) 007 [hep-th/0201028] [INSPIRE].

[114] G. Obied, H. Ooguri, L. Spodyneiko and C. Vafa, de Sitter Space and the Swampland, arXiv:1806.08362 [INSPIRE]. 
[115] H. Ooguri, E. Palti, G. Shiu and C. Vafa, Distance and de Sitter Conjectures on the Swampland, Phys. Lett. B 788 (2019) 180 [arXiv:1810.05506] [INSPIRE].

[116] F. Denef, M.R. Douglas and B. Florea, Building a better racetrack, JHEP 06 (2004) 034 [hep-th/0404257] [INSPIRE].

[117] J.P. Conlon, F. Quevedo and K. Suruliz, Large-volume flux compactifications: Moduli spectrum and D3/D7 soft supersymmetry breaking, JHEP 08 (2005) 007 [hep-th/0505076] [INSPIRE]. 